\title{
SEMICONTINUITY THEOREM IN THE MICROPOLAR ELASTICITY*
}

\author{
Josip TAMBAČA ${ }^{1}$ AND IgOR VELČIĆ ${ }^{1}$
}

\begin{abstract}
In this paper we investigate the equivalence of the sequential weak lower semicontinuity of the total energy functional and the quasiconvexity of the stored energy function of the nonlinear micropolar elasticity. Based on techniques of Acerbi and Fusco [Arch. Rational Mech. Anal. 86 (1984) 125-145] we extend the result from Tambača and Velčić [ESAIM: COCV (2008) DOI: 10.1051/cocv:2008065] for energies that satisfy the growth of order $p \geq 1$. This result is the main step towards the general existence theorem for the nonlinear micropolar elasticity.
\end{abstract}

Mathematics Subject Classification. 74A35, 74G25, 74G65.

Received July 24, 2008.

Published online February 10, 2009.

\section{INTRODUCTION}

In micropolar elasticity, in contrast to the classical elasticity where the motion of a material particle is fully described by a vector function called deformation function $\varphi: \Omega \rightarrow \mathbb{R}^{3}$, we suppose that material particles undergo an additional micromotion, corresponding to the rotation $\overline{\mathbf{R}}: \Omega \rightarrow \mathrm{SO}(3)$ of the material particle at the microscale. Such generalized continua are introduced by the Cosserat brothers in [5]. For the overview of the micropolar elasticity, which is a special case of the microstretch continua see [7]. For the physical relevance of the micropolar (and micromorphic) elasticity in conjunction with finite elasto-plasticity and elastic metallic foams see $[18,21]$.

Existence theorems in the linearized micropolar elasticity are usually based on the uniform positivity of the stored energy function (see [8] or [2]). A new approach has been recently taken by Jeong and Neff in [9] in considering the weakest possible, conformally invariant curvature expression (see [10,22]). In this way, physical inconsistencies present for uniform positive curvature assumptions can be avoided (see [16]). The first existence theorems for geometrically exact Cosserat and micromorphic models, based on convexity arguments are also given by Neff in [17] (micromorphic elasticity is more general theory than micropolar elasticity). Also, for generalized continua with microstructure the existence theorem is given in [11] where convexity in the derivative of the variable which describes microstructure is demanded (in the micropolar case that would mean convexity in $\nabla \overline{\mathbf{R}}$ ). In our work we extend these developments in the micropolar case to more general constitutive behavior.

An approach to the existence theorems in the classical elasticity for general energy is based on the direct methods of the calculus of variations, see [3,6]. This approach is applied in [26] to obtain an existence theorem

Keywords and phrases. Micropolar elasticity, existence theorem, quasiconvexity, semicontinuity.

* This work was supported in part by grant 037-0693014-2765 from the Ministry of Education, Science and Sports of the Republic of Croatia.

1 Department of Mathematics, University of Zagreb, Bijenička 30, 10000 Zagreb, Croatia. tambaca@math.hr; ivelcic@math.hr 
for the nonlinear micropolar elasticity. It is based on the equivalence of the sequential weak lower semicontinuity of the total energy function and the quasiconvexity in $\nabla \boldsymbol{\varphi}$ and $\boldsymbol{\omega}$ (a variable which is introduced in [25] and is related to $\nabla_{i} \overline{\mathbf{R}}$ ). This approach provided, under certain conditions, the equivalence for the stored energy function satisfying the growth condition of order $p$, only for $p>m$, where $m$ is the dimension of the reference configuration $\Omega$ of the micropolar body.

In this paper we apply the techniques of [1] and [6], pp. 367-393, and extend the equivalence for stored energy functions with growth condition in $\nabla \boldsymbol{\varphi}$ of order $r$ and growth condition in $\boldsymbol{\omega}$ of order $p$ for all $p, r \in[1, \infty]$. Therefore quadratic stored energy functions (analogous to the Biot material, see $[4,25]$ ) are now included in the theory. As a direct consequence of the obtained result the existence of a minimizer of the total energy follows. Moreover, we expect to use the result in justification of lower-dimensional models from the three-dimensional equations by means of $\Gamma$-convergence starting from general energy functions. For the derivation and justification of the models for geometrically exact Cosserat plates and shells see $[15,19,20,25]$.

Through the paper we use the notation $\|\cdot\|$ for the norm in the appropriate Euclidean space. As a rule lower subscript denotes the element of a sequence, upper subscript, e.g. $\boldsymbol{R}^{j}$, denotes the $j$ th column of the matrix $\mathbf{R}$ and upper subscripts in brackets, e.g. $R^{(i j)}$, or $R^{(i)}$, denotes the $i, j$ element of the matrix $\mathbf{R}$ or $i$ th element of a vector $\boldsymbol{R}$. By $\mathbf{A}_{\boldsymbol{v}}$ we denote skew-symmetric matrix associated to its axial vector $\boldsymbol{v}$, i.e. $\mathbf{A}_{\boldsymbol{v}} x=\boldsymbol{v} \times x$.

\section{Micropolar elasticity, SEMICONTINUity, QUASICONVEXITY}

Let $\Omega \subset \mathbb{R}^{m}, m=1,2,3$, be an open bounded set with the Lipschitz boundary. The strain energy functional of the homogeneous micropolar body with the reference configuration $\bar{\Omega}$ is given by

$$
I(\boldsymbol{\varphi}, \overline{\mathbf{R}} ; \Omega)=\int_{\Omega} W(\nabla \varphi(x), \overline{\mathbf{R}}(x), \nabla \overline{\mathbf{R}}(x)) \mathrm{d} x,
$$

where $W$ is the stored energy function (i.e. the volume density of the internal energy in the reference configuration). As $\overline{\mathbf{R}}$ is a rotation the matrix $\partial_{i} \overline{\mathbf{R}} \overline{\mathbf{R}}^{T}$ is skew-symmetric. Its axial vector we denote by $\boldsymbol{\omega}^{i}$, i.e.

$$
\mathbf{A}_{\boldsymbol{\omega}^{i}}=\partial_{i} \overline{\mathbf{R}} \overline{\mathbf{R}}^{T}, \quad i=1, \ldots, m,
$$

where the notation $\mathbf{A}_{\boldsymbol{\omega}^{i}}$ stands for the skew-symmetric matrix with the axial vector $\boldsymbol{\omega}^{i}$. This definition is the same as in $[25,26]$ since $\boldsymbol{\omega}^{i}$ then satisfy

$$
\partial_{i} \overline{\mathbf{R}}=\boldsymbol{\omega}^{i} \times \overline{\mathbf{R}}=\mathbf{A}_{\omega^{i}} \overline{\mathbf{R}}, \quad i=1, \ldots, m,
$$

where the vector product is taken with respect to the columns of $\overline{\mathbf{R}}$. Then vectors $\boldsymbol{\omega}^{i}$ can also be expressed by

$$
\boldsymbol{\omega}^{i}=\boldsymbol{\omega}(\overline{\mathbf{R}})^{i}=\frac{1}{2} \overline{\boldsymbol{R}}^{j} \times \partial_{i} \overline{\boldsymbol{R}}^{j}, \quad i=1, \ldots, m,
$$

where $\overline{\mathbf{R}}=\left(\overline{\boldsymbol{R}}^{1} \overline{\boldsymbol{R}}^{2} \overline{\boldsymbol{R}}^{3}\right)$ and the summation convention is used. In the same manner we denote $\boldsymbol{\omega}=$ $\left(\boldsymbol{\omega}^{1} \ldots \boldsymbol{\omega}^{m}\right)$. Now we change the dependence of the stored energy function and assume that the energy functional is given by

$$
I(\boldsymbol{\varphi}, \overline{\mathbf{R}} ; \Omega)=\int_{\Omega} W(\nabla \boldsymbol{\varphi}(x), \overline{\mathbf{R}}(x), \boldsymbol{\omega}(x)) \mathrm{d} x .
$$

Motivation for this change is that, due to $\overline{\mathbf{R}}$ being rotation (pointwise it belongs to the three-dimensional manifold $\mathrm{SO}(3)$ ) derivatives of $\overline{\mathbf{R}}$ are dependent (there are 27 of them). However, $\boldsymbol{\omega}$ have independent components and there is one-to-one, purely algebraic, correspondence between $(\overline{\mathbf{R}}, \partial \overline{\mathbf{R}})$ and $(\overline{\mathbf{R}}, \boldsymbol{\omega})$ for $\overline{\mathbf{R}} \in \operatorname{SO}(3)$. Note as well that there is an analogy between vector columns of $\boldsymbol{\omega}$ and angular velocity. For that change the following Lemma 1.1 is essential. That all $27 \partial_{i} \overline{\mathbf{R}}$ derivatives can be controlled by 9 independent components is obvious 
by the geometry of SO(3). In [23] it is shown that $\overline{\mathbf{R}}^{T}$ Curl $\overline{\mathbf{R}}$ is isomorphic to $\boldsymbol{\omega}$ and suggested as curvature measure. The reason why we work with $\boldsymbol{\omega}$ is the way the oscillations of $\overline{\mathbf{R}}$ affect $\boldsymbol{\omega}$ (see Rem. 2.2).

Lemma 1.1. Let $\Omega \subset \mathbb{R}^{m}$ be a bounded open set and $p \in[1, \infty]$. Let $\overline{\mathbf{R}}_{k}, \overline{\mathbf{R}} \in W^{1, p}(\Omega, \mathrm{SO}(3))$ and let $\boldsymbol{\omega}_{k}^{i}=\boldsymbol{\omega}\left(\overline{\mathbf{R}}_{k}\right)^{i}, \boldsymbol{\omega}^{i}=\boldsymbol{\omega}(\overline{\mathbf{R}})^{i}$. Then $\overline{\mathbf{R}}_{k} \rightarrow \overline{\mathbf{R}}$ in $W^{1, p}\left(\Omega, \mathbb{R}^{3 \times 3}\right)$ if and only if

$$
\overline{\mathbf{R}}_{k} \rightarrow \overline{\mathbf{R}} \text { in } L^{p}\left(\Omega, \mathbb{R}^{3 \times 3}\right) \text { and } \boldsymbol{\omega}_{k}^{i} \rightarrow \boldsymbol{\omega}^{i} \text { in } L^{p}\left(\Omega, \mathbb{R}^{3}\right), i=1, \ldots, m .
$$

Moreover, the same holds for the weak convergence (weak $*$ for $p=\infty$ ).

Proof. See Lemma 2.7 and Remark 2.8 from [26].

Note also that, since $\overline{\mathbf{R}}$ is bounded we can control the norm of $\nabla \overline{\mathbf{R}}$ by the norm $\boldsymbol{\omega}$ and the opposite (i.e. there exist constants $C_{1}, C_{2}>0$, which depend on the vector and matrix norm we choose, such that $\left.C_{1}\left\|\partial_{i} \overline{\mathbf{R}}\right\| \leq\left\|\omega^{i}\right\| \leq C_{2}\left\|\partial_{i} \overline{\mathbf{R}}\right\|\right)$.

In the sequel we discuss the sequential weak lower semicontinuity of the functional $I$. It is important to guarantee that the weak limit of the minimizing sequence is the global minima of the functional i.e. to guarantee the existence of the solution of the minimum of the energy of micropolar body (see Prop. 2.2 in [26]). In the case of the classical elasticity the sequential weak lower semicontinuity of the total energy (under some additional conditions on the stored energy function) is equivalent to the quasiconvexity of the stored energy function in $\nabla \varphi$. Let us recall the definition of the quasiconvex functions.

Definition 1.2. The function $W: \mathbb{R}^{n \times m} \rightarrow \mathbb{R}$ is quasiconvex if it is Borel measurable, locally integrable and satisfies

$$
W(\mathbf{A}) \leq \frac{1}{\operatorname{meas}(D)} \int_{D} W(\mathbf{A}+\nabla \chi(x)) \mathrm{d} x
$$

for every open bounded set $D \subset \mathbb{R}^{m}$ with Lipschitz boundary, for every $\mathbf{A} \in \mathbb{R}^{n \times m}$ and $\chi \in W_{0}^{1, \infty}\left(D, \mathbb{R}^{n}\right)$.

In the last definition $W_{0}^{1, \infty}\left(D, \mathbb{R}^{n}\right)$ is understood in the sense of Meyers, see [12], i.e. a set of $W^{1, \infty}\left(D, \mathbb{R}^{n}\right)$ functions with the zero trace at the boundary; that is different from the closure of $C_{0}^{\infty}\left(D, \mathbb{R}^{n}\right)$ in $W^{1, \infty}\left(D, \mathbb{R}^{n}\right)$ norm.

One should also note that in the definition of quasiconvexity it is enough to demand the property for an arbitrary cube $D$ (see [6], Rem. 5.2, p. 157).

In [26] we have proved the equivalence of the sequential weak lower semicontinuity of the total energy function $I$ and the quasiconvexity of the energy density function $W$ in $\nabla \boldsymbol{\varphi}$ and $\boldsymbol{\omega}$ (the first and third variable) in the case of micropolar elasticity. The technique we have applied provided insight into the problem, but unfortunately we were able to prove the sufficiency result only for $p>m$ which excludes some important energy density functions quadratic in $\nabla \boldsymbol{\varphi}$ or $\boldsymbol{\omega}$. The necessity and sufficiency results proven in [26] are as follows.

Theorem 1.3 (necessity of quasiconvexity). Let $\Omega \subset \mathbb{R}^{m}$ be an open bounded set, let $W: \mathbb{R}^{3 \times m} \times \mathrm{SO}(3) \times$ $\mathbb{R}^{3 \times m} \rightarrow \mathbb{R}$ be continuous and let the functional defined by

$$
I(\boldsymbol{\varphi}, \overline{\mathbf{R}} ; \Omega)=\int_{\Omega} W(\nabla \boldsymbol{\varphi}(x), \overline{\mathbf{R}}(x), \boldsymbol{\omega}(x)) \mathrm{d} x
$$

be sequentially weakly lower semicontinuous, i.e. it satisfies the condition

$$
I(\varphi, \overline{\mathbf{R}} ; \Omega) \leq \liminf _{k \rightarrow \infty} I\left(\varphi_{k}, \overline{\mathbf{R}}_{k} ; \Omega\right)
$$

for every sequence $\left(\left(\boldsymbol{\varphi}_{k}, \overline{\mathbf{R}}_{k}\right)\right)_{k} \subset W^{1, \infty}\left(\Omega ; \mathbb{R}^{3}\right) \times W^{1, \infty}(\Omega ; \mathrm{SO}(3))$ that converges weak $*$ to $(\boldsymbol{\varphi}, \overline{\mathbf{R}})$ in $W^{1, \infty}\left(\Omega ; \mathbb{R}^{3}\right) \times W^{1, \infty}\left(\Omega ; \mathbb{R}^{3 \times 3}\right)$. 
Then $W$ is quasiconvex in the first and the last variable i.e. $W$ satisfies

$$
W(\mathbf{A}, \overline{\mathbf{R}}, \mathbf{B}) \leq \frac{1}{\operatorname{meas}(D)} \int_{D} W(\mathbf{A}+\nabla \chi(x), \overline{\mathbf{R}}, \mathbf{B}+\nabla \boldsymbol{\psi}(x)) \mathrm{d} x
$$

for every open bounded set $D$ with Lipschitz boundary, for every $\mathbf{A}, \mathbf{B} \in \mathbb{R}^{3 \times m}, \overline{\mathbf{R}} \in \mathrm{SO}(3)$ and for every $\chi, \boldsymbol{\psi} \in W_{0}^{1, \infty}\left(D, \mathbb{R}^{3}\right)$.

Since weak* ${ }^{*}$ convergence is stronger than weak convergence in any $W^{1, p}$ this theorem also implies that quasiconvexity of energy density function is necessary for sequential weak lower continuity with respect to $W^{1, p}$.

Theorem 1.4 (sufficiency of quasiconvexity). Let $\Omega \subset \mathbb{R}^{m}$ be an open bounded set with the Lipschitz boundary and $m<p<\infty$. Let $W: \mathbb{R}^{3 \times m} \times \mathrm{SO}(3) \times \mathbb{R}^{3 \times m} \rightarrow \mathbb{R}$ be quasiconvex in the first and the last variable and satisfies (a), (b), (c), (d) below. Let

$$
I(\boldsymbol{\varphi}, \overline{\mathbf{R}} ; \Omega)=\int_{\Omega} W(\nabla \boldsymbol{\varphi}(x), \overline{\mathbf{R}}(x), \boldsymbol{\omega}(x)) \mathrm{d} x .
$$

Then for every sequence $\left(\left(\boldsymbol{\varphi}_{k}, \overline{\mathbf{R}}_{k}\right)\right)_{k} \subset W^{1, p}\left(\Omega, \mathbb{R}^{3}\right) \times W^{1, p}(\Omega, \operatorname{SO}(3))$ which converges weakly to $(\boldsymbol{\varphi}, \overline{\mathbf{R}})$ in $W^{1, p}\left(\Omega, \mathbb{R}^{3}\right) \times W^{1, p}\left(\Omega, \mathbb{R}^{3 \times 3}\right)$ one has

$$
I(\varphi, \overline{\mathbf{R}} ; \Omega) \leq \liminf _{k} I\left(\varphi_{k}, \overline{\mathbf{R}}_{k} ; \Omega\right)
$$

The conditions (a), (b), (c), (d) are given by

(a) $W(\mathbf{A}, \overline{\mathbf{R}}, \mathbf{B}) \leq K_{g}\left(1+\|\mathbf{A}\|^{p}+\|\mathbf{B}\|^{p}\right), \quad \mathbf{A}, \mathbf{B} \in \mathbb{R}^{3 \times m}, \overline{\mathbf{R}} \in \mathrm{SO}(3)$;

(b) $\left|W\left(\mathbf{A}_{1}, \overline{\mathbf{R}}, \mathbf{B}_{1}\right)-W\left(\mathbf{A}_{2}, \overline{\mathbf{R}}, \mathbf{B}_{2}\right)\right| \leq K_{g}\left(1+\left\|\mathbf{A}_{1}\right\|^{p-1}+\left\|\mathbf{B}_{1}\right\|^{p-1}+\left\|\mathbf{A}_{2}\right\|^{p-1}+\left\|\mathbf{B}_{2}\right\|^{p-1}\right)\left(\left\|\mathbf{A}_{1}-\mathbf{A}_{2}\right\|+\right.$ $\left.\left\|\mathbf{B}_{1}-\mathbf{B}_{2}\right\|\right)$,

$\mathbf{A}_{1}, \mathbf{A}_{2}, \mathbf{B}_{1}, \mathbf{B}_{2} \in \mathbb{R}^{3 \times m}, \overline{\mathbf{R}} \in \mathrm{SO}(3)$;

(c) $W \geq-\beta$, for some $\beta \geq 0$;

(d) $\left|W\left(\mathbf{A}, \overline{\mathbf{R}}_{1}, \mathbf{B}\right)-W\left(\mathbf{A}, \overline{\mathbf{R}}_{2}, \mathbf{B}\right)\right| \leq K_{g}\left(1+\|\mathbf{A}\|^{p}+\|\mathbf{B}\|^{p}\right)\left\|\overline{\mathbf{R}}_{1}-\overline{\mathbf{R}}_{2}\right\|$,

$\mathbf{A}, \mathbf{B} \in \mathbb{R}^{3 \times m}, \overline{\mathbf{R}}_{1}, \overline{\mathbf{R}}_{2} \in \mathrm{SO}(3)$.

Additionally we have proved that for $p=\infty$ the conditions (a), (b), (c) and (d) are not necessary (see Rem. 4.7 in [26]). Moreover, the conditions (b) and (d) for general $p$ can be replaced by objectivity, quasiconvexity and the condition (a) (see Prop. 4.9 in [26]).

In the following section we improve Theorem 1.4 in three directions. First we show that the condition $p>m$ is not necessary. Second, we show that the conditions (b) and (d) are also not necessary (without imposing objectivity). Third, we allow the growth condition in $\nabla \varphi$ and $\boldsymbol{\omega}$ to be different, allowing greater class of stored energy functions. The proof is technically involved and uses techniques adapted from Acerbi and Fusco [1]. Using Theorem 2.13 we can restate Theorem 2.5 from [26] as follows.

Theorem 1.5. Let $\Omega \subset \mathbb{R}^{m}$ be an open bounded set with the Lipschitz boundary and p,r $\in\langle 1, \infty\rangle$. Let $W: \mathbb{R}^{3 \times m} \times \mathrm{SO}(3) \times \mathbb{R}^{3 \times m} \rightarrow \mathbb{R}$ be a quasiconvex in the first and the last variable (see Thm. 1.3 for the definition) and objective function which satisfies

(a) (growth condition) $W(\mathbf{A}, \overline{\mathbf{R}}, \mathbf{B}) \leq K\left(1+\|\mathbf{A}\|^{r}+\|\mathbf{B}\|^{p}\right), \quad \mathbf{A}, \mathbf{B} \in \mathbb{R}^{3 \times m}, \overline{\mathbf{R}} \in \mathrm{SO}(3)$;

(b) (coercivity) there exist $C_{1}>0$ and $C_{2} \in \mathbb{R}$ such that

$$
W(\mathbf{A}, \overline{\mathbf{R}}, \mathbf{B}) \geq C_{1}\left(\|\mathbf{A}\|^{r}+\|\mathbf{B}\|^{p}\right)+C_{2}, \quad \mathbf{A}, \mathbf{B} \in \mathbb{R}^{3 \times m}, \overline{\mathbf{R}} \in \operatorname{SO}(3) .
$$

Then the total energy functional $I^{*}$ defined by

$$
I^{*}(\boldsymbol{\varphi}, \overline{\mathbf{R}})=\int_{\Omega}\left(W(\nabla \boldsymbol{\varphi}, \overline{\mathbf{R}}, \boldsymbol{\omega})-\Pi_{\boldsymbol{f}}(\boldsymbol{\varphi})-\Pi_{\mathbf{M}}(\overline{\mathbf{R}})\right) \mathrm{d} V-\int_{\Gamma_{S}} \Pi_{\boldsymbol{n}}(\boldsymbol{\varphi}) \mathrm{d} S-\int_{\Gamma_{C}} \Pi_{\mathbf{M}_{c}}(\overline{\mathbf{R}}) \mathrm{d} S
$$


attains its minimum in the set

$$
\Phi=\left\{(\boldsymbol{\varphi}, \overline{\mathbf{R}}) \in W^{1, r}\left(\Omega, \mathbb{R}^{3}\right) \times W^{1, p}(\Omega, \mathrm{SO}(3)),\left.\varphi\right|_{\Gamma}=\boldsymbol{g}_{d},\left.\overline{\mathbf{R}}\right|_{\Gamma}=\overline{\mathbf{R}}_{d}\right\},
$$

provided that $\Gamma$ is a part of the boundary with non-zero measure and the set $\Phi$ is non-empty. Here $\Pi_{\boldsymbol{f}}, \Pi_{\mathbf{M}}$, $\Pi_{\boldsymbol{n}}, \Pi_{\mathbf{M}_{c}}$ are potentials of external loads and $\Gamma_{S}, \Gamma_{C}, \Gamma$ are parts of boundary, $\boldsymbol{g}_{d}: \Gamma \rightarrow \mathbb{R}^{3}, \overline{\mathbf{R}}_{d}: \Gamma \rightarrow \mathrm{SO}(3)$ (see [14] or [26] for details).

Remark 1.6. If we introduce a simple isotropic quadratic stored energy function (as treated e.g. in [14]) of the type $\left(\overline{\mathbf{U}}=\overline{\mathbf{R}}^{T} \nabla \boldsymbol{\varphi}-\right.$ first Cosserat deformation tensor see [18])

$$
W(\nabla \boldsymbol{\varphi}, \overline{\mathbf{R}}, \boldsymbol{\omega})=\mu\|\operatorname{sym} \overline{\mathbf{U}}-\mathbf{I}\|^{2}+\mu_{c}\|\operatorname{skew} \overline{\mathbf{U}}\|^{2}+\frac{\lambda}{2} \operatorname{tr}[\overline{\mathbf{U}}-\mathbf{I}]^{2}+\mu L_{c}^{p}\|\boldsymbol{\omega}\|^{p}
$$

we conclude that the coerciveness assumption in Theorem 1.5 would imply $\mu_{c}>0$. This is undesirable property since there are some physical situations where $\mu_{c}=0$ is a reasonable demand (see [18]). However, in the existence proof the coerciveness is needed just to conclude that the minimizing sequence is bounded. Therefore we can deal with this situation like in [17], using extended three dimensional Korn's inequality proved in [24] (which improves the result in [13]). For this we need $p>3$. Thus we have the existence result for this energy as well (which can also be proved by convexity arguments, see [17]) for any $p>1$ if $\mu_{c}>0$ and for any $p>3$ if $\mu_{c}=0$. The case $\mu_{c}=0$ and $1 \leq p \leq 3$ (called the "fracture case" by Neff) remains open.

\section{The MAIN THEOREM}

In this section we formulate and prove the sufficiency of quasiconvexity of the energy density function theorem. We do it in two steps. In the first step we prove that the convergence (1.1) holds for a perturbations of rotation by equiintegrable sequence of rotations (Thm. 2.4). In the second step we extend it on all perturbations (Thm. 2.13).

By direct calculation we obtain the following lemma.

Lemma 2.1. Let $\overline{\mathbf{R}}_{k}, \overline{\mathbf{R}}: \Omega \rightarrow \mathrm{SO}(3)$ smooth enough. Then

$$
\boldsymbol{\omega}_{k}^{i}=\boldsymbol{\omega}^{i}+\partial_{i}\left(\frac{1}{2} \overline{\boldsymbol{R}}^{j} \times \overline{\boldsymbol{R}}_{k}^{j}\right)+\left(\overline{\boldsymbol{R}}_{k}^{j}-\overline{\boldsymbol{R}}^{j}\right) \times \partial_{i} \overline{\boldsymbol{R}}^{j}+\frac{1}{2}\left(\overline{\boldsymbol{R}}_{k}^{j}-\overline{\boldsymbol{R}}^{j}\right) \times \partial_{i}\left(\overline{\boldsymbol{R}}_{k}^{j}-\overline{\boldsymbol{R}}^{j}\right),
$$

for $i=1,2,3$.

Remark 2.2. If we suppose that $\left(\overline{\mathbf{R}}_{k}\right)_{k} \subset W^{1, p}(\Omega, \mathrm{SO}(3))$ and $\overline{\mathbf{R}}_{k} \rightarrow \overline{\mathbf{R}} \in W^{1, p}\left(\Omega, \mathbb{R}^{3 \times 3}\right)$, Lemma 2.1 tells us how a weakly convergent sequence of rotations changes $\boldsymbol{\omega}_{k}=\boldsymbol{\omega}\left(\overline{\mathbf{R}}_{k}\right)$ in the neighborhood of its limit $\boldsymbol{\omega}=\boldsymbol{\omega}(\overline{\mathbf{R}})$. Essentially, $\boldsymbol{\omega}_{k}$ are equal to $\boldsymbol{\omega}+\nabla \boldsymbol{\zeta}$ where $\boldsymbol{\zeta} \rightarrow 0$ in $W^{1, p}\left(\Omega, \mathbb{R}^{3}\right)$ (the other parts can be shown to be not important for the sequential weak lower semicontinuity of the functional $I$, since they converge to 0 strongly in $L^{p}$ on an arbitrarily subset $\Omega_{1} \subset \Omega$ ). This establishes the analogy between $\boldsymbol{\omega}$ and $\nabla \varphi$ for the deformation $\varphi$ since $\nabla \varphi_{k}=\nabla \varphi+\nabla\left(\varphi_{k}-\varphi\right)$. This is the key observation which justifies the use of the variable $\boldsymbol{\omega}$ in answering the question of sequential weak lower semicontinuity of the functional $I$.

By direct adaption of the proof of Lemma 8.7. from [6], p. 371, we conclude the following lemma.

Lemma 2.3. Let $D \subset \mathbb{R}^{m}$ be a cube parallel to the axes, $p, r \in[1, \infty], W: \mathbb{R}^{n \times m} \times \mathbb{R}^{k \times m} \rightarrow \mathbb{R}$ be quasiconvex, i.e. it is Borel measurable and locally integrable function which satisfies

$$
W(\mathbf{A}, \mathbf{B}) \leq \frac{1}{\operatorname{meas}(D)} \int_{D} W(\mathbf{A}+\nabla \chi(x), \mathbf{B}+\nabla \boldsymbol{\psi}(x)) \mathrm{d} x,
$$


for every open bounded set $D \subset \mathbb{R}^{m}$ with Lipschitz boundary, for every $\mathbf{A} \in \mathbb{R}^{n \times m}, \mathbf{B} \in \mathbb{R}^{k \times m}$ and $\chi \in$ $W_{0}^{1, \infty}\left(D, \mathbb{R}^{n}\right), \boldsymbol{\psi} \in W_{0}^{1, \infty}\left(D, \mathbb{R}^{k}\right)$, and also satisfies

- if $p, r \in[1, \infty\rangle$

$$
-\beta \leq W(\mathbf{A}, \mathbf{B}) \leq K_{g}\left(1+\|\mathbf{A}\|^{r}+\|\mathbf{B}\|^{p}\right) ;
$$

- if $p=\infty, r \in[1, \infty\rangle$

$$
-\beta \leq W(\mathbf{A}, \mathbf{B}) \leq \eta(\|\mathbf{B}\|)\left(1+\|\mathbf{A}\|^{r}\right),
$$

where $\eta$ is a continuous and increasing function;

- if $p \in[1, \infty\rangle, r=\infty$

$$
W(\mathbf{A}, \mathbf{B}) \leq \eta(\|\mathbf{A}\|)\left(1+\|\mathbf{B}\|^{p}\right),
$$

where $\eta$ is a continuous and increasing function;

- if $p=\infty, r=\infty$

$$
|W(\mathbf{A}, \mathbf{B})| \leq \eta(\|\mathbf{A}\|,\|\mathbf{B}\|),
$$

where $\eta$ is a continuous and increasing functions in each of its arguments (if $W$ is continuous this is satisfied).

Then we have

$$
W(\mathbf{A}, \mathbf{B}) \operatorname{meas}(D) \leq \liminf _{k \rightarrow \infty} \int_{D} W\left(\mathbf{A}+\nabla \boldsymbol{\chi}_{k}(x), \mathbf{B}+\nabla \boldsymbol{\psi}_{k}(x)\right) \mathrm{d} x
$$

for every $\mathbf{A} \in \mathbb{R}^{n \times m}, \mathbf{B} \in \mathbb{R}^{k \times m}$ and $\boldsymbol{\chi}_{k} \rightarrow 0$ weakly in $W^{1, r}\left(D ; \mathbb{R}^{n}\right)$ (weak ${ }^{*}$ if $\left.r=\infty\right), \boldsymbol{\psi}_{k} \rightarrow 0$ weakly in $W^{1, p}\left(D ; \mathbb{R}^{k}\right)($ weak $*$ if $p=\infty)$.

Proof. Let $D^{0} \subset D$ be a cube and let $R=\frac{1}{2} \operatorname{dist}\left(D^{0}, D\right)$. Let $M \in \mathbb{N}$ and let $D^{0} \subset D^{m} \subset D, 1 \leq m \leq M$ be such that

$$
\operatorname{dist}\left(D^{0}, \partial D^{m}\right)=\frac{m}{M} R, \quad 1 \leq m \leq M .
$$

We choose $\varphi^{m} \in C^{\infty}(D), 1 \leq m \leq M$ such that

$$
\left\{\begin{array}{l}
0 \leq \varphi^{m} \leq 1 \\
\varphi^{m}(x)=\left\{\begin{aligned}
1 & \text { if } x \in D^{m-1} \\
0 & \text { if } x \in D \backslash D^{m}
\end{aligned}\right. \\
\left|\nabla \varphi^{m}\right| \leq a \frac{M}{R},
\end{array}\right.
$$

where $a>0$ is a constant. Let

$$
\boldsymbol{\chi}_{k}^{m}=\varphi^{m} \boldsymbol{\chi}_{k}, \quad \boldsymbol{\psi}_{k}^{m}=\varphi^{m} \boldsymbol{\psi}_{k}
$$

Then $\boldsymbol{\chi}_{k}^{m}, \boldsymbol{\psi}_{k}^{m}=0$ on $\partial D$ so we may use the quasiconvexity of $W$ to get

$$
\begin{aligned}
\int_{D} W(\mathbf{A}, \mathbf{B}) \mathrm{d} x \leq & \int_{D} W\left(\mathbf{A}+\nabla \boldsymbol{\chi}_{k}^{m}(x), \mathbf{B}+\nabla \boldsymbol{\psi}_{k}^{m}(x)\right) \mathrm{d} x \\
\leq & \int_{D \backslash D^{m}} W(\mathbf{A}, \mathbf{B}) \mathrm{d} x+\int_{D^{m} \backslash D^{m-1}} W\left(\mathbf{A}+\nabla \boldsymbol{\chi}_{k}^{m}(x), \mathbf{B}+\nabla \boldsymbol{\psi}_{k}^{m}(x)\right) \mathrm{d} x \\
& +\int_{D^{m-1}} W\left(\mathbf{A}+\nabla \boldsymbol{\chi}_{k}(x), \mathbf{B}+\nabla \boldsymbol{\psi}_{k}(x)\right) \mathrm{d} x
\end{aligned}
$$

We then deduce that

$$
\int_{D^{m}} W(\mathbf{A}, \mathbf{B}) \mathrm{d} x \leq \int_{D^{m} \backslash D^{m-1}} W\left(\mathbf{A}+\nabla \boldsymbol{\chi}_{k}^{m}(x), \mathbf{B}+\nabla \boldsymbol{\psi}_{k}^{m}(x)\right) \mathrm{d} x+\int_{D^{m-1}} W\left(\mathbf{A}+\nabla \boldsymbol{\chi}_{k}(x), \mathbf{B}+\nabla \boldsymbol{\psi}_{k}(x)\right) \mathrm{d} x .
$$


We may also rewrite the above inequality in the following way

$$
\begin{aligned}
\int_{D^{m}} W(\mathbf{A}, \mathbf{B}) \mathrm{d} x \leq & \int_{D} W\left(\mathbf{A}+\nabla \boldsymbol{\chi}_{k}(x), \mathbf{B}+\nabla \boldsymbol{\psi}_{k}(x)\right) \mathrm{d} x \\
& -\int_{D \backslash D^{m-1}} W\left(\mathbf{A}+\nabla \boldsymbol{\chi}_{k}(x), \mathbf{B}+\nabla \boldsymbol{\psi}_{k}(x)\right) \mathrm{d} x \\
& +\int_{D^{m} \backslash D^{m-1}} W\left(\mathbf{A}+\nabla \boldsymbol{\chi}_{k}^{m}(x), \mathbf{B}+\nabla \boldsymbol{\psi}_{k}^{m}(x)\right) \mathrm{d} x \\
= & \int_{D} W\left(\mathbf{A}+\nabla \boldsymbol{\chi}_{k}(x), \mathbf{B}+\nabla \boldsymbol{\psi}_{k}(x)\right) \mathrm{d} x+\alpha_{1}+\alpha_{2} .
\end{aligned}
$$

By choosing $R$ sufficiently small, since $W$ is bounded below, we have that

$$
\alpha_{1} \leq \varepsilon
$$

We estimate $\alpha_{2}$

$$
\alpha_{2}=\int_{D^{m} \backslash D^{m-1}} W\left(\mathbf{A}+\nabla \boldsymbol{\chi}_{k}^{m}(x), \mathbf{B}+\nabla \boldsymbol{\psi}_{k}^{m}(x)\right) \mathrm{d} x
$$

for $p, r \in[1, \infty\rangle$. Cases $p=\infty, r \in[1, \infty\rangle$ and $r=\infty, p \in[1, \infty\rangle$ proceed similarly. The case $p=\infty, r=\infty$, using the boundness of sequences $\left\|\nabla \boldsymbol{\chi}_{k}^{m}\right\|_{L^{\infty}},\left\|\nabla \boldsymbol{\psi}_{k}^{m}\right\|_{L^{\infty}}$ is simple.

Let $\alpha$ denotes a generic constant. Then for $p, r \in[1, \infty\rangle$ we estimate

$$
\begin{aligned}
\alpha_{2} & \leq \alpha \int_{D^{m} \backslash D^{m-1}}\left(1+\left\|\mathbf{A}+\nabla \boldsymbol{\chi}_{k}^{m}(x)\right\|^{r}+\left\|\mathbf{B}+\nabla \boldsymbol{\psi}_{k}^{m}(x)\right\|^{p}\right) \mathrm{d} x \\
& \leq \alpha \int_{D^{m} \backslash D^{m-1}}\left(1+\|\mathbf{A}\|^{r}+\left\|\varphi^{m} \nabla \boldsymbol{\chi}_{k}+\boldsymbol{\chi}_{k} \nabla \varphi^{m}\right\|^{r}+\|\mathbf{B}\|^{p}+\left\|\varphi^{m} \nabla \boldsymbol{\psi}_{k}+\boldsymbol{\psi}_{k} \nabla \varphi^{m}\right\|^{p}\right) \mathrm{d} x \\
& \leq \alpha \int_{D^{m} \backslash D^{m-1}}\left(1+\|\mathbf{A}\|^{r}+\left\|\nabla \boldsymbol{\chi}_{k}\right\|^{r}+\left(\frac{a M}{R}\right)^{r}\left\|\boldsymbol{\chi}_{k}\right\|^{r}+\|\mathbf{B}\|^{p}+\left\|\nabla \boldsymbol{\psi}_{k}\right\|^{p}+\left(\frac{a M}{R}\right)^{p}\left\|\boldsymbol{\psi}_{k}\right\|^{p}\right) \mathrm{d} x .
\end{aligned}
$$

Summing (2.3) for $m=1$ to $M$ and using (2.4) and (2.5) we have

$$
\begin{aligned}
M \int_{D} W(\mathbf{A} & \left.+\boldsymbol{\chi}_{k}(x), \mathbf{B}+\boldsymbol{\psi}_{k}(x)\right) \mathrm{d} x-W(\mathbf{A}, \mathbf{B})\left(\sum_{m=1}^{M} \operatorname{meas} D^{m}\right) \geq \\
& -M \varepsilon-\alpha \int_{D^{M} \backslash D^{0}}\left(1+\|\mathbf{A}\|^{r}+\left\|\nabla \boldsymbol{\chi}_{k}\right\|^{r}+\left(\frac{a M}{R}\right)^{r}\left\|\boldsymbol{\chi}_{k}\right\|^{r}+\|\mathbf{B}\|^{p}+\left\|\nabla \boldsymbol{\psi}_{k}\right\|^{p}+\left(\frac{a M}{R}\right)^{p}\left\|\boldsymbol{\psi}_{k}\right\|^{p}\right) \mathrm{d} x .
\end{aligned}
$$

Dividing the above inequality by $M$ and letting $k \rightarrow \infty$, using Sobolev imbedding and recalling that $\chi_{k} \rightarrow 0$ in $W^{1, r}\left(D ; \mathbb{R}^{n}\right)$ and $\boldsymbol{\psi}_{k} \rightarrow 0$ in $W^{1, p}\left(D ; \mathbb{R}^{k}\right)$, we have that

$$
\liminf _{k \rightarrow \infty} \int_{D} W\left(\mathbf{A}+\chi_{k}(x), \mathbf{B}+\boldsymbol{\psi}_{k}(x)\right) \mathrm{d} x-W(\mathbf{A}, \mathbf{B})\left(\frac{1}{M} \sum_{m=1}^{M} \operatorname{meas} D^{m}\right) \geq-\varepsilon-\frac{\gamma}{M},
$$

where $\gamma$ is a constant. Letting $m \rightarrow \infty$, taking into account the arbitrariness of $D^{0}$ and $\varepsilon$ we obtain the statement of the theorem.

In the following theorem we prove that the semicontinuity (1.1) holds for equiintegrable sequences adapting the proof of Lemma 8.14 in [6], p. 384. 
Theorem 2.4. Let $\Omega \subset \mathbb{R}^{m}$ be an arbitrary bounded open set and $p, r \in[1, \infty\rangle$. Let $W: \mathbb{R}^{3 \times m} \times \mathrm{SO}(3) \times \mathbb{R}^{3 \times m} \rightarrow$ $\mathbb{R}$ be continuous, quasiconvex in the first and last variable and

(1) $W(\mathbf{A}, \overline{\mathbf{R}}, \mathbf{B}) \geq-\beta, \quad \mathbf{A}, \mathbf{B} \in \mathbb{R}^{3 \times m}, \overline{\mathbf{R}} \in \mathrm{SO}(3)$;

(2) $W(\mathbf{A}, \overline{\mathbf{R}}, \mathbf{B}) \leq K_{g}\left(1+\|\mathbf{A}\|^{r}+\|\mathbf{B}\|^{p}\right), \quad \mathbf{A}, \mathbf{B} \in \mathbb{R}^{3 \times m}, \overline{\mathbf{R}} \in \mathrm{SO}(3)$.

Let $\boldsymbol{\varphi} \in W^{1, r}\left(\Omega, \mathbb{R}^{3}\right),\left(\overline{\mathbf{R}}_{k}\right)_{k} \subset W^{1, p}(\Omega, \mathrm{SO}(3))$. Let $\boldsymbol{\varphi}_{k} \rightarrow \boldsymbol{\varphi} \in W^{1, r}\left(\Omega, \mathbb{R}^{3}\right), \overline{\mathbf{R}}_{k} \rightarrow \overline{\mathbf{R}} \in W^{1, p}\left(\Omega, \mathbb{R}^{3 \times 3}\right)$. Moreover, let the sequences of $L^{1}(\Omega)$ functions $\left(\left\|\nabla \boldsymbol{\varphi}_{k}\right\|^{r}\right)_{k}$ and $\left(\left\|\boldsymbol{\omega}_{k}\right\|^{p}\right)_{k}$ be equiintegrable (this is not a condition for $r=1$ or $p=1)$. Let $I$ be the functional defined on $W^{1, r}\left(\Omega, \mathbb{R}^{3}\right) \times W^{1, p}(\Omega, \mathrm{SO}(3))$ by

$$
I(\boldsymbol{\varphi}, \overline{\mathbf{R}} ; \Omega)=\int_{\Omega} W(\nabla \varphi, \overline{\mathbf{R}}, \boldsymbol{\omega}) .
$$

Then

$$
\liminf _{k \rightarrow \infty} I\left(\boldsymbol{\varphi}_{k}, \overline{\mathbf{R}}_{k} ; \Omega\right) \geq I(\boldsymbol{\varphi}, \overline{\mathbf{R}} ; \Omega) .
$$

Proof. Without loss of generality we can assume $\beta=0$ (otherwise we prove the theorem for $W+\beta$ ). First we take $\Omega=\langle 0,1\rangle^{m}$. There exists a subsequence $\left(\left(\boldsymbol{\varphi}_{k_{n}}, \overline{\mathbf{R}}_{k_{n}}\right)\right)_{n}$ such that

$$
\liminf _{k \rightarrow \infty} I\left(\boldsymbol{\varphi}_{k}, \overline{\mathbf{R}}_{k} ; \Omega\right)=\lim _{n \rightarrow \infty} I\left(\boldsymbol{\varphi}_{k_{n}}, \overline{\mathbf{R}}_{k_{n}} ; \Omega\right)
$$

In the sequel we consider this subsequence only and denote it by $\left(\left(\varphi_{k}, \overline{\mathbf{R}}_{k}\right)\right)_{k}$. Because of the equiintegrability there is a function $\eta: \mathbb{R}^{+} \rightarrow \mathbb{R}^{+}$which is continuous nondecreasing such that $\eta(0)=0$ and for every measurable $B \subset \Omega$ one has

$$
\int_{B}\left(1+\|\nabla \boldsymbol{\varphi}(x)\|^{r}+\left\|\nabla \boldsymbol{\varphi}_{k}(x)\right\|^{r}+\|\boldsymbol{\omega}(x)\|^{p}+\left\|\boldsymbol{\omega}_{k}(x)\right\|^{p}\right) \mathrm{d} x \leq \eta(\operatorname{meas}(B)) .
$$

Let $\varepsilon>0$. Then there exists $\alpha(\varepsilon) \geq 1$ such that for

$$
E_{\varepsilon}=\left\{x \in \Omega:\|\nabla \varphi(x)\|^{r} \leq \alpha(\varepsilon) \text { and }\|\boldsymbol{\omega}(x)\|^{p} \leq \alpha(\varepsilon)\right\}
$$

one has

$$
\operatorname{meas}\left(\Omega \backslash E_{\varepsilon}\right)<\varepsilon, \quad \int_{\Omega \backslash E_{\varepsilon}}\left(\|\nabla \varphi(x)\|^{r}+\|\boldsymbol{\omega}(x)\|^{p}\right) \mathrm{d} x<\varepsilon .
$$

We also take $K_{\varepsilon} \subset \Omega$, a compact set such that meas $\left(\Omega \backslash K_{\varepsilon}\right) \leq \frac{\varepsilon}{\alpha(\varepsilon)}$ and that (the representatives of) $\overline{\mathbf{R}}, \nabla \boldsymbol{\varphi}, \boldsymbol{\omega}$ are continuous on $K_{\varepsilon}$ and $\overline{\mathbf{R}}_{k} \rightarrow \overline{\mathbf{R}}$ strongly in $L^{\infty}\left(K_{\varepsilon}, \mathbb{R}^{3 \times 3}\right)$; this can be done by Lusin's and Egoroff's theorem. Because of the boundedness of $\left(\nabla \boldsymbol{\varphi}_{k}\right)_{k}$ and $\left(\boldsymbol{\omega}_{k}\right)_{k}$ in $L^{r}(\Omega)$ i.e. $L^{p}(\Omega)$, using Chebyshev's inequality, there exists $M(\varepsilon)$ (independent of $k$ ) such that meas $\left(\Omega \backslash H_{k}^{\varepsilon}\right)<\frac{\varepsilon}{\alpha(\varepsilon)}$ where

$$
H_{k}^{\varepsilon}=\left\{x \in \Omega:\left\|\nabla \boldsymbol{\varphi}_{k}(x)\right\| \leq M(\varepsilon) \text { and }\left\|\boldsymbol{\omega}_{k}(x)\right\| \leq M(\varepsilon)\right\} .
$$

Using Lemma 2.1 we obtain

$$
\boldsymbol{\omega}_{k}(x)=\boldsymbol{\omega}(x)+\nabla\left(\frac{1}{2} \overline{\boldsymbol{R}}^{j}(x) \times \overline{\mathbf{R}}_{k}^{j}(x)\right)+\boldsymbol{s}_{k}(x) \text { a.e. in } \Omega,
$$


where $\left\|\boldsymbol{s}_{k}\right\|_{L^{\infty}\left(K_{\varepsilon} \cap H_{k}^{\varepsilon}, \mathbb{R}^{3 \times m}\right)} \rightarrow 0$. Let us define

$$
\psi_{k}(x)=\frac{1}{2} \overline{\mathbf{R}}^{j}(x) \times \overline{\mathbf{R}}_{k}^{j}(x) .
$$

Note that for some $C_{\psi}>1$ we have

$$
\left\|\nabla \boldsymbol{\psi}_{k}(x)\right\|^{p} \leq C_{\boldsymbol{\psi}}\left(\|\boldsymbol{\omega}(x)\|^{p}+\left\|\boldsymbol{\omega}_{k}(x)\right\|^{p}\right) \quad \text { a.e. in } \Omega, \quad k \in \mathbb{N} .
$$

We divide $\Omega$ in open cubes $Q_{h}^{n}$ of length size $2^{-n}$ such that

$$
\Omega=\bigcup_{h=1}^{2^{n m}} Q_{h}^{n} \cup Z,
$$

where $Z$ is of measure zero. For all $n, h$ such that meas $\left(Q_{h}^{n} \cap K_{\varepsilon} \cap E_{\varepsilon}\right) \neq 0$ let us choose $x_{h}^{n} \in Q_{h}^{n} \cap K_{\varepsilon} \cap E_{\varepsilon}$ such that $\overline{\mathbf{R}}\left(x_{h}^{n}\right)$ is a rotation (see Rem. 2.5). Then

$$
I\left(\boldsymbol{\varphi}_{k}, \overline{\mathbf{R}}_{k} ; \Omega\right) \geq I\left(\boldsymbol{\varphi}_{k}, \overline{\mathbf{R}}_{k} ; K_{\varepsilon} \cap E_{\varepsilon} \cap H_{k}^{\varepsilon}\right)=I_{1}+I_{2}+I_{3}+I_{4}+I_{5},
$$

where

$$
\begin{aligned}
I_{1}= & \int_{K_{\varepsilon} \cap E_{\varepsilon} \cap H_{k}^{\varepsilon}}\left[W\left(\nabla \boldsymbol{\varphi}_{k}(x), \overline{\mathbf{R}}_{k}(x), \boldsymbol{\omega}_{k}(x)\right)-W\left(\nabla \boldsymbol{\varphi}_{k}(x), \overline{\mathbf{R}}(x), \boldsymbol{\omega}(x)+\nabla \boldsymbol{\psi}_{k}(x)\right)\right] \mathrm{d} x, \\
I_{2}= & \sum_{h} \int_{Q_{h}^{n} \cap K_{\varepsilon} \cap E_{\varepsilon} \cap H_{k}^{\varepsilon}}\left[W\left(\nabla \boldsymbol{\varphi}(x)+\nabla\left(\boldsymbol{\varphi}_{k}-\boldsymbol{\varphi}\right)(x), \overline{\mathbf{R}}(x), \boldsymbol{\omega}(x)+\nabla \boldsymbol{\psi}_{k}(x)\right)\right. \\
& \left.-W\left(\nabla \boldsymbol{\varphi}\left(x_{h}^{n}\right)+\nabla\left(\boldsymbol{\varphi}_{k}-\boldsymbol{\varphi}\right)(x), \overline{\mathbf{R}}\left(x_{h}^{n}\right), \boldsymbol{\omega}\left(x_{h}^{n}\right)+\nabla \boldsymbol{\psi}_{k}(x)\right)\right] \mathrm{d} x, \\
I_{3}= & \sum_{h} \int_{Q_{h}^{n} \cap K_{\varepsilon} \cap E_{\varepsilon} \cap H_{k}^{\varepsilon}}\left[W\left(\nabla \boldsymbol{\varphi}\left(x_{h}^{n}\right)+\nabla\left(\boldsymbol{\varphi}_{k}-\boldsymbol{\varphi}\right)(x), \overline{\mathbf{R}}\left(x_{h}^{n}\right), \boldsymbol{\omega}\left(x_{h}^{n}\right)+\nabla \boldsymbol{\psi}_{k}(x)\right)\right. \\
& \left.-W\left(\nabla \boldsymbol{\varphi}\left(x_{h}^{n}\right), \overline{\mathbf{R}}\left(x_{h}^{n}\right), \boldsymbol{\omega}\left(x_{h}^{n}\right)\right)\right] \mathrm{d} x, \\
I_{4}= & \sum_{h} \int_{Q_{h}^{n} \cap K_{\varepsilon} \cap E_{\varepsilon} \cap H_{k}^{\varepsilon}}\left[W\left(\nabla \boldsymbol{\varphi}\left(x_{h}^{n}\right), \overline{\mathbf{R}}\left(x_{h}^{n}\right), \boldsymbol{\omega}\left(x_{h}^{n}\right)\right)-W(\nabla \boldsymbol{\varphi}(x), \overline{\mathbf{R}}(x), \boldsymbol{\omega}(x))\right] \mathrm{d} x, \\
I_{5}= & I\left(\boldsymbol{\varphi}, \overline{\mathbf{R}}, K_{\varepsilon} \cap E_{\varepsilon} \cap H_{k}^{\varepsilon}\right) .
\end{aligned}
$$

Sequences $\left(\nabla \boldsymbol{\varphi}_{k}\right)_{k},\left(\boldsymbol{\omega}_{k}\right)_{k},\left(\nabla \boldsymbol{\psi}_{k}\right)_{k}$ are bounded in $L^{\infty}\left(K_{\varepsilon} \cap H_{k}^{\varepsilon}\right)$. Therefore the arguments of $W$ in $I_{1}$ and $I_{2}$ belong to the compact set. Applying the uniform continuity of $W$ we conclude $\lim _{k \rightarrow \infty} I_{1}=0$ (recall that $\left.\left\|\boldsymbol{s}_{k}\right\|_{L^{\infty}\left(K_{\varepsilon} \cap H_{k}^{\varepsilon}, \mathbb{R}^{3 \times m}\right)} \rightarrow 0\right)$. Using also that $\nabla \boldsymbol{\varphi}, \overline{\mathbf{R}}, \boldsymbol{\omega}$ are uniformly continuous on $K_{\varepsilon}$ we conclude that $\lim _{n \rightarrow \infty} I_{2}=\lim _{n \rightarrow \infty} I_{4}=0$, uniformly by $k$ (note that difference in the arguments of $W$ in this case is independent of $k$ ). Therefore we take $n$ large enough such that $I_{2}+I_{4} \leq \varepsilon$. 
Now we estimate $I_{3}$. We first estimate the integral over $Q_{h}^{n} \backslash\left(K_{\varepsilon} \cap E_{\varepsilon} \cap H_{k}^{\varepsilon}\right)$

$$
\begin{aligned}
& \left|\sum_{h} \int_{Q_{h}^{n} \backslash\left(K_{\varepsilon} \cap E_{\varepsilon} \cap H_{k}^{\varepsilon}\right)}\left[W\left(\nabla \boldsymbol{\varphi}\left(x_{h}^{n}\right)+\nabla\left(\boldsymbol{\varphi}_{k}-\boldsymbol{\varphi}\right)(x), \overline{\mathbf{R}}\left(x_{h}^{n}\right), \boldsymbol{\omega}\left(x_{h}^{n}\right)+\nabla \boldsymbol{\psi}_{k}(x)\right)-W\left(\nabla \boldsymbol{\varphi}\left(x_{h}^{n}\right), \overline{\mathbf{R}}\left(x_{h}^{n}\right), \boldsymbol{\omega}\left(x_{h}^{n}\right)\right)\right] \mathrm{d} x\right| \\
& \leq \sum_{h} \int_{\left(\left(Q_{h}^{n} \backslash K_{\varepsilon}\right) \cup\left(Q_{h}^{n} \backslash H_{k}^{\varepsilon}\right)\right)}\left|W\left(\nabla \boldsymbol{\varphi}\left(x_{h}^{n}\right)+\nabla\left(\boldsymbol{\varphi}_{k}-\boldsymbol{\varphi}\right)(x), \overline{\mathbf{R}}\left(x_{h}^{n}\right), \boldsymbol{\omega}\left(x_{h}^{n}\right)+\nabla \boldsymbol{\psi}_{k}(x)\right)\right| \\
& \quad+\sum_{h} \int_{\left(\left(Q_{h}^{n} \backslash K_{\varepsilon}\right) \cup\left(Q_{h}^{n} \backslash H_{k}^{\varepsilon}\right)\right)}\left|W\left(\nabla \boldsymbol{\varphi}\left(x_{h}^{n}\right), \overline{\mathbf{R}}\left(x_{h}^{n}\right), \boldsymbol{\omega}\left(x_{h}^{n}\right)\right)\right| \mathrm{d} x \\
& \quad+\sum_{h} \int_{Q_{h}^{n} \backslash E_{\varepsilon}}\left|W\left(\nabla \boldsymbol{\varphi}\left(x_{h}^{n}\right)+\nabla\left(\boldsymbol{\varphi}_{k}-\boldsymbol{\varphi}\right)(x), \overline{\mathbf{R}}\left(x_{h}^{n}\right), \boldsymbol{\omega}\left(x_{h}^{n}\right)+\nabla \boldsymbol{\psi}_{k}(x)\right)\right| \\
& \quad+\sum_{h} \int_{Q_{h}^{n} \backslash E_{\varepsilon}}\left|W\left(\nabla \boldsymbol{\varphi}\left(x_{h}^{n}\right), \overline{\mathbf{R}}\left(x_{h}^{n}\right), \boldsymbol{\omega}\left(x_{h}^{n}\right)\right)\right| \mathrm{d} x \\
& =J_{1}+J_{2}+J_{3}+J_{4} .
\end{aligned}
$$

Using (2.8) and the growth condition we conclude

$$
\begin{aligned}
\left|J_{1}\right| & \leq \sum_{h} \int_{\left(\left(Q_{h}^{n} \backslash K_{\varepsilon}\right) \cup\left(Q_{h}^{n} \backslash H_{k}^{\varepsilon}\right)\right)} K^{\prime}\left(1+\left\|\nabla \boldsymbol{\varphi}\left(x_{h}^{n}\right)\right\|^{r}+\| \nabla\left(\boldsymbol{\varphi}_{k}(x)\left\|^{r}+\right\| \nabla \boldsymbol{\varphi}(x)\left\|^{r}+\right\| \boldsymbol{\omega}\left(x_{h}^{n}\right)\left\|^{p}+\right\| \nabla \boldsymbol{\psi}_{k}(x) \|^{p}\right) \mathrm{d} x\right. \\
& \leq 4 K^{\prime} \varepsilon+K^{\prime} C_{\boldsymbol{\psi}} \eta(2 \varepsilon),
\end{aligned}
$$

In the similar way we conclude

Now we estimate $J_{3}$

$$
\left|J_{2}\right| \leq 6 K_{g} \varepsilon
$$

$$
\begin{aligned}
\left|J_{3}\right| & \leq K^{\prime} \sum_{h} \int_{Q_{h}^{n} \backslash E_{\varepsilon}}\left(1+\left\|\nabla \boldsymbol{\varphi}\left(x_{h}^{n}\right)\right\|^{r}+\left\|\nabla \boldsymbol{\varphi}_{k}(x)\right\|^{r}+\|\nabla \boldsymbol{\varphi}(x)\|^{r}+\left\|\boldsymbol{\omega}\left(x_{h}^{n}\right)\right\|^{p}+\left\|\nabla \boldsymbol{\psi}_{k}(x)\right\|^{p}\right) \mathrm{d} x \\
& \leq K^{\prime} \eta(\varepsilon)+2 K^{\prime} \int_{\Omega \backslash E_{\varepsilon}} \alpha(\varepsilon) \leq K^{\prime} \eta(\varepsilon)+2 K^{\prime} \int_{\Omega \backslash E_{\varepsilon}}\left(\|\nabla \boldsymbol{\varphi}(x)\|^{r}+\|\boldsymbol{\omega}(x)\|^{p}\right) \leq 3 K^{\prime} \eta(\varepsilon) .
\end{aligned}
$$

In the similar way

Thus using Lemma 2.3 we have

$$
\left|J_{4}\right| \leq 3 K_{g} \varepsilon
$$

$$
\liminf _{k \rightarrow \infty} I_{3} \geq \sum_{h} \liminf _{k \rightarrow \infty} \int_{Q_{h}^{n}} \cdot-\sum_{h} \limsup _{k \rightarrow \infty} \int_{Q_{h}^{n} \backslash\left(K_{\varepsilon} \cap E_{\varepsilon} \cap H_{k}^{\varepsilon}\right)} \cdot \geq \liminf _{k \rightarrow \infty} \int_{Q_{h}^{n}} \cdot-O(\varepsilon) \geq-O(\varepsilon)
$$

where $\lim _{\varepsilon \rightarrow 0} O(\varepsilon)=0$. Finally, in the same manner we estimate

$$
I_{5} \leq K_{g} \int_{\Omega \backslash\left(K_{\varepsilon} \cap E_{\varepsilon} \cap H_{k}^{\varepsilon}\right)}\left(1+\|\boldsymbol{\varphi}(x)\|^{r}+\|\boldsymbol{\omega}(x)\|^{r}\right) \mathrm{d} x \leq K_{g} \eta\left(\operatorname{meas}\left(\Omega \backslash\left(K_{\varepsilon} \cap E_{\varepsilon} \cap H_{k}^{\varepsilon}\right)\right)\right) \leq K_{g} \eta(3 \varepsilon),
$$

which can be written as

therefore

$$
I_{5}=I\left(\boldsymbol{\varphi}, \overline{\mathbf{R}} ; K_{\varepsilon} \cap E_{\varepsilon} \cap H_{k}^{\varepsilon}\right) \geq I(\boldsymbol{\varphi}, \overline{\mathbf{R}} ; \Omega)-K_{g} \eta(3 \varepsilon),
$$

$$
\liminf _{k \rightarrow \infty} I\left(\boldsymbol{\varphi}_{k}, \overline{\mathbf{R}}_{k} ; \Omega\right) \geq I(\boldsymbol{\varphi}, \overline{\mathbf{R}} ; \Omega)-O(\varepsilon) .
$$

Since $\varepsilon$ can be arbitrarily small we have the claim. 
Now we have to prove the claim for an arbitrary bounded open $\Omega$. First, we can prove the claim for any cube in the same way as above. From the properties of the Lebesgue integral we have

$$
I(\boldsymbol{\varphi}, \overline{\mathbf{R}} ; \Omega)=\sup \left\{\sum_{l=0}^{n} I\left(\boldsymbol{\varphi}, \overline{\mathbf{R}} ; D_{l}\right): n \in \mathbb{N}, D_{l} \subset \Omega, D_{l} \text { disjoint open cubes }\right\} .
$$

The property (2.6), because it holds for every $I\left(\boldsymbol{\varphi}, \overline{\mathbf{R}}, D_{k}\right)$, also holds for every sum. Moreover (2.6) holds for the supremum of an arbitrary set of functionals if it holds for every functional in that set, so we have the claim.

Remark 2.5. In Theorem 2.4 we have chosen $x_{h}^{n}$ and then $\nabla \boldsymbol{\varphi}\left(x_{h}^{n}\right), \overline{\mathbf{R}}\left(x_{h}^{n}\right), \boldsymbol{\omega}\left(x_{h}^{n}\right)$. Of course that this does not make sense for classes of functions $\nabla \boldsymbol{\varphi}, \overline{\mathbf{R}}, \boldsymbol{\omega}$ equal just almost everywhere. It should be read that we take some special representants (continuous on $K_{\varepsilon}$ ) whose existence is guaranteed by Lusin's theorem and then choose $\nabla \boldsymbol{\varphi}_{h}^{n}:=\nabla \boldsymbol{\varphi}\left(x_{h}^{n}\right), \overline{\mathbf{R}}_{h}^{n}:=\overline{\mathbf{R}}\left(x_{h}^{n}\right), \boldsymbol{\omega}_{h}^{n}:=\boldsymbol{\omega}\left(x_{h}^{n}\right)$ (we must also choose $x_{h}^{n}$ such that $\overline{\mathbf{R}}\left(x_{h}^{n}\right)$ is a rotation which is possible if meas $\left(Q_{h}^{n} \cap K_{\varepsilon}\right)>0$ ). These values are good approximations of every representant in the class $\nabla \varphi$ i.e. $\overline{\mathbf{R}}$ i.e. $\boldsymbol{\omega}$ in the set $Q_{h}^{n} \cap K_{\varepsilon}$ in the sense that for $n$ large enough and every $h$ we have that $\left\|\nabla \varphi(x)-\nabla \varphi_{h}^{n}\right\| \leq \varepsilon$ a.e. in $Q_{h}^{n} \cap K_{\varepsilon}$ i.e. $\left\|\overline{\mathbf{R}}(x)-\overline{\mathbf{R}}_{h}^{n}\right\| \leq \varepsilon$ a.e. in $Q_{h}^{n} \cap K_{\varepsilon}$ i.e. $\left\|\boldsymbol{\omega}(x)-\boldsymbol{\omega}_{h}^{n}\right\| \leq \varepsilon$ a.e. in $Q_{h}^{n} \cap K_{\varepsilon}$.

Remark 2.6. The statement of Theorem 2.4 holds for $p, r \in[1, \infty]$ under the assumptions of Lemma 2.3. When $p=\infty$ and $r=\infty$ the proof is direct, without the construction of the sets $K_{\varepsilon}, E_{\varepsilon}, H_{\varepsilon}^{k}$. When just one of them is infinity, we proceed defining $K_{\varepsilon}, E_{\varepsilon}, H_{\varepsilon}^{k}$ in the same as in the proof using the sequence, $\left\|\nabla \varphi_{k}\right\|$ or $\left\|\boldsymbol{\omega}_{k}\right\|$, which is not uniformly bounded.

The following four lemmas will be useful. The proofs are given in [1].

Lemma 2.7. Let $G \subset \mathbb{R}^{m}$ be a measurable set such that meas $(G)<\infty$. Assume $\left(M_{k}\right)_{k}$ is a sequence of measurable subsets of $G$ such that for some $\varepsilon>0$ holds

$$
\operatorname{meas}\left(M_{k}\right) \geq \varepsilon \quad k \in \mathbb{N} .
$$

Then there exists a subsequence $\left(M_{k_{h}}\right)_{h}$ such that $\bigcap_{h \in \mathbb{N}} M_{k_{h}} \neq \emptyset$.

Lemma 2.8. Let $\left(\phi_{k}\right)_{k} \subset L^{1}\left(\mathbb{R}^{m}\right)$ be bounded. Then for every $\varepsilon>0$ there exists $\left(A_{\varepsilon}, \delta, S\right)$ where $A_{\varepsilon}$ is measurable and meas $\left(A_{\varepsilon}\right)<\varepsilon, \delta>0$, and $S$ is an infinite subset of $\mathbb{N}$, such that for all $k \in S$

$$
\int_{B}\left|\phi_{k}(x)\right| \mathrm{d} x<\varepsilon
$$

whenever $B$ and $A_{\varepsilon}$ are disjoint and meas $(B)<\delta$.

Let $r>0$ and $x \in \mathbb{R}^{n}$. We denote $B_{r}(x)=\left\{y \in \mathbb{R}^{m}:\|y-x\|<r\right\}$. Then meas $\left(B_{r}(x)\right)=C(m) r^{m}$, where $C(m)$ is the volume of the unit ball in $\mathbb{R}^{m}$.

Definition 2.9. Let $u \in C_{0}^{\infty}\left(\mathbb{R}^{m}\right)$. We define

$$
\left(M^{*} u\right)(x)=(M u)(x)+\sum_{i=1}^{m}\left(M \partial_{i} u\right)(x),
$$

where

$$
(M u)(x)=\sup _{r>0} \frac{1}{C(m) r^{m}} \int_{B_{r}(x)}|u(y)| \mathrm{d} y .
$$


Lemma 2.10. If $u \in C_{0}^{\infty}\left(\mathbb{R}^{m}\right)$ then $M^{*} u \in C^{0}\left(\mathbb{R}^{m}\right)$ and

$$
|u(x)|+\sum_{i=1}^{m}\left|\partial_{i} u(x)\right| \leq\left(M^{*} u\right)(x)
$$

for every $x \in \mathbb{R}^{m}$. Moreover, if $p>1$, then there is $c(m, p)>0$ such that

$$
\left\|M^{*} u\right\|_{L^{p}\left(\mathbb{R}^{m}\right)} \leq c(m, p)\|u\|_{W^{1, p}\left(\mathbb{R}^{m}\right)}
$$

and if $p=1$ then there is $c(m)>0$ such that for all $\lambda>0$ one has

$$
\operatorname{meas}\left\{x \in \mathbb{R}^{m}:\left(M^{*} u\right) \geq \lambda\right\} \leq \frac{c(m)}{\lambda}\|u\|_{W^{1,1}\left(\mathbb{R}^{m}\right)} .
$$

Lemma 2.11. Let $u \in C_{0}^{\infty}\left(\mathbb{R}^{m}\right), \lambda>0$ and

$$
H^{\lambda}=\left\{x \in \mathbb{R}^{m}:\left(M^{*} u\right)(x)<\lambda\right\} .
$$

Then for every $x, y \in H^{\lambda}$ we have

$$
\frac{|u(y)-u(x)|}{|y-x|} \leq c(m) \lambda .
$$

By expanding the exponential function in the Taylor series we obtain the following lemma.

Lemma 2.12. Let $\Omega \subset \mathbb{R}^{m}$ be an open bounded set with the Lipschitz boundary and $p \in[1, \infty\rangle$. Let $\overline{\mathbf{R}} \in$ $W^{1, p}(\Omega, \mathrm{SO}(3))$ and $\boldsymbol{\psi}_{k}, \boldsymbol{\psi} \in W^{1, \infty}\left(\Omega, \mathbb{R}^{3}\right)$ such that $\boldsymbol{\psi}_{k} \rightarrow \boldsymbol{\psi}$ strongly in $L^{\infty}\left(\Omega, \mathbb{R}^{3}\right)$. Then

$$
\exp \left(\mathbf{A}_{\psi_{k}}\right) \overline{\mathbf{R}} \rightarrow \exp \left(\mathbf{A}_{\psi}\right) \overline{\mathbf{R}} \text { strongly in } L^{\infty}\left(\Omega, \mathbb{R}^{3 \times 3}\right) .
$$

Additionally, if $\boldsymbol{\psi}_{k} \rightarrow \boldsymbol{\psi}$ weak* in $W^{1, \infty}\left(\Omega, \mathbb{R}^{3}\right)$ then:

(a) $\exp \left(\mathbf{A}_{\psi_{k}}\right) \rightarrow \exp \left(\mathbf{A}_{\psi}\right)$ weak $^{*}$ in $W^{1, \infty}\left(\Omega, \mathbb{R}^{3 \times 3}\right)$;

(b) $\exp \left(\mathbf{A}_{\psi_{k}}\right) \overline{\mathbf{R}} \rightarrow \exp \left(\mathbf{A}_{\psi}\right) \overline{\mathbf{R}}$ strongly in $L^{\infty}\left(\Omega, \mathbb{R}^{3 \times 3}\right)$;

(c) $\exp \left(\mathbf{A}_{\psi_{k}}\right) \overline{\mathbf{R}} \rightarrow \exp \left(\mathbf{A}_{\psi}\right) \overline{\mathbf{R}}$ weakly in $W^{1, p}\left(\Omega, \mathbb{R}^{3 \times 3}\right)$.

In the following theorem we prove the statement of Theorem 2.4 without imposing the condition of equiintegrability. The idea behind it is to approximate sequences $\left(\varphi_{k}\right)_{k},\left(\overline{\mathbf{R}}_{k}\right)_{k}$, such that $\boldsymbol{\varphi}_{k} \rightarrow \boldsymbol{\varphi}, \overline{\mathbf{R}}_{k} \rightarrow \overline{\mathbf{R}}$ by equiintegrable sequences $\left(\hat{\boldsymbol{\varphi}}_{k}\right)_{k},\left(\hat{\mathbf{R}}_{k}\right)_{k}$ which satisfy for arbitrary small $\varepsilon$ :

(1) $\liminf _{k \rightarrow \infty} I\left(\boldsymbol{\varphi}_{k}, \overline{\mathbf{R}}_{k}\right) \geq \liminf _{k \rightarrow \infty} I\left(\hat{\boldsymbol{\varphi}}_{k}, \hat{\mathbf{R}}_{k}\right)-O(\varepsilon)$

(2) $\hat{\varphi}_{k} \rightarrow \hat{\varphi}, \hat{\mathbf{R}}_{k} \rightarrow \hat{\mathbf{R}}$

(3) $\operatorname{meas}\{\boldsymbol{\varphi} \neq \hat{\boldsymbol{\varphi}}$ or $\overline{\mathbf{R}} \neq \hat{\mathbf{R}}\}=O(\varepsilon)$.

The claim then follows by applying Theorem 2.4. The same construction was applied in [1]. The novelty here is the approximations of rotations from Lemma 2.12 i.e. $\hat{\mathbf{R}}_{k}=\exp \left(\mathbf{A}_{\boldsymbol{\psi}_{k}}\right) \overline{\mathbf{R}}$, where $\boldsymbol{\psi}_{k} \rightarrow \boldsymbol{\psi}$ weak* in $W^{1, \infty}\left(\Omega, \mathbb{R}^{3}\right)$ and $\operatorname{meas}\{\boldsymbol{\psi} \neq 0\}=O(\varepsilon)$.

Theorem 2.13. Let $p, r \in\langle 1, \infty\rangle$ and $\Omega \subset \mathbb{R}^{m}$ be an arbitrary open bounded set. Let $W: \mathbb{R}^{3 \times m} \times \mathrm{SO}(3) \times$ $\mathbb{R}^{3 \times m} \rightarrow \mathbb{R}$ be continuous, quasiconvex in the first and last variable which satisfies

(1) $W(\mathbf{A}, \overline{\mathbf{R}}, \mathbf{B}) \geq-\beta, \quad \mathbf{A}, \mathbf{B} \in \mathbb{R}^{3 \times m}, \overline{\mathbf{R}} \in \mathrm{SO}(3)$;

(2) $W(\mathbf{A}, \overline{\mathbf{R}}, \mathbf{B}) \leq K_{g}\left(1+\|\mathbf{A}\|^{r}+\|\mathbf{B}\|^{p}\right), \quad \mathbf{A}, \mathbf{B} \in \mathbb{R}^{3 \times m}, \overline{\mathbf{R}} \in \mathrm{SO}(3)$.

Let $\boldsymbol{\varphi}_{k}, \boldsymbol{\varphi} \in W^{1, r}\left(\Omega, \mathbb{R}^{3}\right), \overline{\mathbf{R}}_{k}, \overline{\mathbf{R}} \in W^{1, p}(\Omega, \mathrm{SO}(3)), \boldsymbol{\varphi}_{k} \rightarrow \boldsymbol{\varphi}$ weakly in $W^{1, r}\left(\Omega, \mathbb{R}^{3}\right)$ and $\overline{\mathbf{R}}_{k} \rightarrow \overline{\mathbf{R}}$ weakly in $W^{1, p}\left(\Omega, \mathbb{R}^{3 \times 3}\right)$. Then we have

$$
I(\varphi, \overline{\mathbf{R}} ; \Omega) \leq \liminf _{k \rightarrow \infty} I\left(\boldsymbol{\varphi}_{k}, \overline{\mathbf{R}}_{k} ; \Omega\right) .
$$


Proof. Without loss of generality we can assume that $\beta=0$ (otherwise we prove the statement for $W+\beta$ ). We shall prove the theorem for $\Omega=\langle 0,1\rangle^{m}$. Then for an arbitrary open $\Omega$ one can follow the same reasoning as in Theorem 2.4. For an arbitrary sequence $\left(\left(\boldsymbol{\varphi}_{k}, \overline{\mathbf{R}}_{k}\right)\right)_{k}$ let us choose a subsequence $\left(\left(\boldsymbol{\varphi}_{k_{n}}, \overline{\mathbf{R}}_{k_{n}}\right)\right)_{n}$ such that

$$
\liminf _{k \rightarrow \infty} I\left(\boldsymbol{\varphi}_{k}, \overline{\mathbf{R}}_{k} ; \Omega\right)=\lim _{n \rightarrow \infty} I\left(\boldsymbol{\varphi}_{k_{n}}, \overline{\mathbf{R}}_{k_{n}} ; \Omega\right)
$$

In the sequel we consider only this subsequence and denote it by $\left(\left(\boldsymbol{\varphi}_{k}, \overline{\mathbf{R}}_{k}\right)\right)_{k}$.

For $\varepsilon>0$ we define

$$
\eta(\varepsilon)=\sup \left\{\int_{B}\left(1+\|\nabla \varphi(x)\|^{r}+\|\boldsymbol{\omega}(x)\|^{p}\right) \mathrm{d} x: B \subset \Omega \text { measurable, meas }(B) \leq \varepsilon\right\} .
$$

Then $\eta: \mathbb{R}^{+} \rightarrow \mathbb{R}^{+}$is continuous nondecreasing function such that $\eta(0)=0$ and for every measurable $B \subset \Omega$ one has

$$
\int_{B}\left(1+\|\nabla \boldsymbol{\varphi}(x)\|^{r}+\|\boldsymbol{\omega}(x)\|^{p}\right) \mathrm{d} x \leq \eta(\operatorname{meas}(B)) .
$$

Let, for each $k \in \mathbb{N}, \widetilde{\boldsymbol{\varphi}}_{k} \in W^{1, r}\left(\mathbb{R}^{m}, \mathbb{R}^{3}\right)$ denotes the extension of $\boldsymbol{\varphi}_{k}$ and let $\left(\boldsymbol{z}_{k}\right)_{k} \subset C_{0}^{\infty}\left(\mathbb{R}^{m}, \mathbb{R}^{3}\right)$ be such that

$$
\left\|\boldsymbol{z}_{k}-\widetilde{\varphi}_{k}\right\|_{W^{1, r}\left(\mathbb{R}^{m}, \mathbb{R}^{3}\right)} \leq \frac{1}{k} \quad k \in \mathbb{N}
$$

Let $\widetilde{\mathbf{R}}_{k}, \widetilde{\mathbf{R}} \in W^{1, p}\left(\mathbb{R}^{m}, \mathbb{R}^{3 \times 3}\right)$ be the extensions of $\overline{\mathbf{R}}_{k}, \overline{\mathbf{R}}$. Let $\left(\mathbf{M}_{k}\right)_{k},\left(\mathbf{S}_{k}\right)_{k} \subset C_{0}^{\infty}\left(\mathbb{R}^{m}, \mathbb{R}^{3 \times 3}\right)$ be such that

$$
\left\|\mathbf{M}_{k}-\widetilde{\mathbf{R}}_{k}\right\|_{W^{1, p}\left(\mathbb{R}^{m}, \mathbb{R}^{3 \times 3}\right)} \leq \frac{1}{k}, \quad\left\|\mathbf{S}_{k}-\widetilde{\mathbf{R}}\right\|_{W^{1, p}\left(\mathbb{R}^{m}, \mathbb{R}^{3 \times 3}\right)} \leq \frac{1}{k}, \quad k \in \mathbb{N} .
$$

Then there is $C(\Omega)>0$ such that

$$
\begin{gathered}
\left\|\widetilde{\mathbf{R}}_{k}\right\|_{L^{\infty}\left(\mathbb{R}^{m}, \mathbb{R}^{3 \times 3}\right)} \leq C(\Omega), \quad\|\widetilde{\mathbf{R}}(x)\|_{L^{\infty}\left(\mathbb{R}^{m}, \mathbb{R}^{3 \times 3}\right)} \leq C(\Omega), \\
\left\|\mathbf{M}_{k}\right\|_{L^{\infty}\left(\mathbb{R}^{m}, \mathbb{R}^{3 \times 3}\right)} \leq C(\Omega), \quad\left\|\mathbf{S}_{k}\right\|_{L^{\infty}\left(\mathbb{R}^{m}, \mathbb{R}^{3 \times 3}\right)} \leq C(\Omega) .
\end{gathered}
$$

Let us fix $\varepsilon>0$. By Lusin's, Egoroff's and Sobolev's embedding theorem there is a compact $K_{\varepsilon} \subset \Omega$ such that meas $\left(\Omega \backslash K_{\varepsilon}\right) \leq \varepsilon$, (the representatives of) $\overline{\mathbf{R}}, \boldsymbol{\varphi}, \boldsymbol{\omega}$ are continuous on $K_{\varepsilon}$ and

(1) $\varphi_{k}-\varphi \rightarrow 0$ strongly in $L^{\infty}\left(K_{\varepsilon}, \mathbb{R}^{3}\right)$;

(2) $\boldsymbol{z}_{k}-\boldsymbol{\varphi}_{k} \rightarrow 0$ strongly in $L^{\infty}\left(K_{\varepsilon}, \mathbb{R}^{3}\right), \nabla \boldsymbol{z}_{k}-\nabla \boldsymbol{\varphi}_{k} \rightarrow 0$ strongly in $L^{\infty}\left(K_{\varepsilon}, \mathbb{R}^{3 \times m}\right)$;

(3) $\overline{\mathbf{R}}_{k} \rightarrow \overline{\mathbf{R}}$ strongly in $L^{\infty}\left(K_{\varepsilon}, \mathbb{R}^{3 \times 3}\right)$;

(4) $\mathbf{S}_{k} \rightarrow \overline{\mathbf{R}}$ strongly in $L^{\infty}\left(K_{\varepsilon}, \mathbb{R}^{3 \times 3}\right), \partial_{i} \mathbf{S}_{k} \rightarrow \partial_{i} \overline{\mathbf{R}}$ strongly in $L^{\infty}\left(K_{\varepsilon}, \mathbb{R}^{3 \times 3}\right)$, for $i=1, \ldots, m$;

(5) $\mathbf{M}_{k}-\overline{\mathbf{R}}_{k} \rightarrow 0$ strongly in $L^{\infty}\left(K_{\varepsilon}, \mathbb{R}^{3 \times 3}\right), \partial_{i} \mathbf{M}_{k}-\partial_{i} \overline{\mathbf{R}}_{k} \rightarrow 0$ strongly in $L^{\infty}\left(K_{\varepsilon}, \mathbb{R}^{3 \times 3}\right)$, for $i=1, \ldots, m$.

Using Lemma 2.8 for every of 21 sequences $\left(M^{*} z_{k}^{(i)}\right)^{r}, i=1,2,3$ and $\left(M^{*} M_{k}^{(i j)}\right)^{p},\left(M^{*} S_{k}^{(i j)}\right)^{p}, i, j=1,2,3$ we can choose subsequences still denoted by $\left(\boldsymbol{z}_{k}\right),\left(\mathbf{M}_{k}\right),\left(\mathbf{S}_{k}\right)$, subset $A_{\varepsilon} \subset \Omega$ meas $\left(A_{\varepsilon}\right)<\varepsilon$ and a real number $\delta>0$ such that

$$
\int_{B}\left[\left(M^{*} z_{k}^{(i)}\right)(x)\right]^{r} \mathrm{~d} x<\varepsilon, \int_{B}\left[\left(M^{*} M_{k}^{(i j)}\right)(x)\right]^{p} \mathrm{~d} x<\varepsilon, \int_{B}\left[\left(M^{*} S_{k}^{(i j)}\right)(x)\right]^{p} \mathrm{~d} x<\varepsilon
$$

for every $k \in \mathbb{N}, i, j=1,2,3$ and $B \subset \Omega \backslash A_{\varepsilon}$ such that meas $(B)<\delta$. By Lemma 2.10 we have the boundedness of the above sequences in $L^{r}(\Omega), L^{p}(\Omega)$ and $L^{p}(\Omega)$, respectively. By Chebyshev's inequality we can choose 
$\lambda_{1}(\varepsilon)>0, \lambda_{2}(\varepsilon)>0$ such that $\lambda_{1}(\varepsilon)^{r}=\lambda_{2}(\varepsilon)^{p}$ and

$$
\begin{aligned}
& \text { meas }\left\{x \in \mathbb{R}^{m}:\left(M^{*} z_{k}^{(i)}\right)(x) \geq \lambda_{1}(\varepsilon)\right\}<\min (\varepsilon, \delta), \\
& \text { meas }\left\{x \in \mathbb{R}^{m}:\left(M^{*} M_{k}^{(i j)}\right)(x) \geq \lambda_{2}(\varepsilon)\right\}<\min (\varepsilon, \delta), \\
& \text { meas }\left\{x \in \mathbb{R}^{m}:\left(M^{*} S_{k}^{(i j)}\right)(x) \geq \lambda_{2}(\varepsilon)\right\}<\min (\varepsilon, \delta),
\end{aligned}
$$

for all $k \in \mathbb{N}, i, j=1,2,3$. We set

$$
\begin{aligned}
H_{i, k}^{\lambda_{1}(\varepsilon)} & =\left\{x \in \mathbb{R}^{m}:\left(M^{*} z_{k}^{(i)}\right)<\lambda_{1}(\varepsilon)\right\} \\
J_{i j, k}^{\lambda_{2}(\varepsilon)} & =\left\{x \in \mathbb{R}^{m}:\left(M^{*} M_{k}^{(i j)}\right)<\lambda_{2}(\varepsilon)\right\} \\
L_{i j, k}^{\lambda_{2}(\varepsilon)} & =\left\{x \in \mathbb{R}^{m}:\left(M^{*} S_{k}^{(i j)}\right)<\lambda_{2}(\varepsilon)\right\}
\end{aligned}
$$

for all $k \in \mathbb{N}, i, j=1,2,3$ and define

$$
H_{k}^{\varepsilon}=\bigcap_{i=1}^{3} H_{i, k}^{\lambda_{1}(\varepsilon)} \bigcap_{i, j=1}^{3} J_{i j, k}^{\lambda_{2}(\varepsilon)} \bigcap_{i, j=1}^{3} L_{i j, k}^{\lambda_{2}(\varepsilon)}, \quad k \in \mathbb{N} .
$$

By Lemma 2.11 there is a constant $c(m)>0$ such that for all $x, y \in H_{k}^{\lambda(\varepsilon)}$ and all $i, j=1,2,3$ one has

$$
\begin{aligned}
& \frac{\left|z_{k}^{(i)}(x)-z_{k}^{(i)}(y)\right|}{|y-x|} \leq c(m) \lambda_{1}(\varepsilon), \\
& \frac{\left|M_{k}^{(i j)}(x)-M_{k}^{(i j)}(y)\right|}{|y-x|} \leq c(m) \lambda_{2}(\varepsilon), \\
& \frac{\left|S_{k}^{(i j)}(x)-S_{k}^{(i j)}(y)\right|}{|y-x|} \leq c(m) \lambda_{2}(\varepsilon) .
\end{aligned}
$$

Let $g_{k}^{(i)}, N_{k}^{(i j)}, T_{k}^{(i j)}$ be the Lipschitz extensions of $z_{k}^{(i)}, M_{k}^{(i j)}, S_{k}^{(i j)}$ outside $H_{k}^{\varepsilon}$ with the Lipschitz constant not greater than $c(m) \lambda_{1}(\varepsilon)$ i.e. $c(m) \lambda_{2}(\varepsilon)$. Because $H_{k}^{\varepsilon}$ is an open set we have

$$
\begin{array}{cl}
g_{k}^{(i)}(x)=z_{k}^{(i)}(x), & \nabla g_{k}^{(i)}(x)=\nabla z_{k}^{(i)}(x), \\
N_{k}^{(i j)}(x)=M_{k}^{(i j)}(x), & \nabla N_{k}^{(i j)}(x)=\nabla M_{k}^{(i j)}(x), \\
T_{k}^{(i j)}(x)=S_{k}^{(i j)}(x), & \nabla T_{k}^{(i j)}(x)=\nabla S_{k}^{(i j)}(x),
\end{array}
$$

for all $x \in H_{k}^{\varepsilon}$. Also we can assume (if not cutting the Lipschitz function we obtain the Lipschitz function with the same constant) that

$$
\begin{aligned}
\left\|g_{k}^{(i)}\right\|_{L^{\infty}\left(\mathbb{R}^{m}\right)} & \leq\left\|z_{k}^{(i)}\right\|_{L^{\infty}\left(H_{k}^{\varepsilon}\right)} \leq \lambda_{1}(\varepsilon), \\
\left\|N_{k}^{(i j)}\right\|_{L^{\infty}\left(\mathbb{R}^{m}\right)} & \leq\left\|M_{k}^{(i j)}\right\|_{L^{\infty}\left(H_{k}^{\varepsilon}\right)} \leq C(\Omega), \\
\left\|T_{k}^{(i j)}\right\|_{L^{\infty}\left(\mathbb{R}^{m}\right)} & \leq\left\|S_{k}^{(i j)}\right\|_{L^{\infty}\left(H_{k}^{\varepsilon}\right)} \leq C(\Omega) .
\end{aligned}
$$


Therefore, at least on a subsequence, one has

$$
\begin{aligned}
& g_{k}^{(i)} \rightarrow g^{(i)} \text { weak* }^{*} \text { in } W^{1, \infty}(\Omega), \\
& N_{k}^{(i j)} \rightarrow N^{(i j)} \text { weak* in } W^{1, \infty}(\Omega), \\
& T_{k}^{(i j)} \rightarrow T^{(i j)} \text { weak* in } W^{1, \infty}(\Omega)
\end{aligned}
$$

for all $i, j=1,2,3$. We now define $\boldsymbol{g}_{k}=\left(g_{k}^{(i)}\right)_{i=1,2,3}, \mathbf{N}_{k}=\left(N_{k}^{(i j)}\right)_{i, j=1,2,3}, \mathbf{T}_{k}=\left(T_{k}^{(i j)}\right)_{i, j=1,2,3}, \boldsymbol{g}=\left(g^{(i)}\right)_{i=1,2,3}$, $\mathbf{N}=\left(N^{(i j)}\right)_{i, j=1,2,3}, \mathbf{T}=\left(T^{(i j)}\right)_{i, j=1,2,3}$ and

$$
\boldsymbol{\psi}_{k}=\frac{1}{2} \boldsymbol{T}_{k}^{j} \times \boldsymbol{N}_{k}^{j}, \quad \boldsymbol{\psi}=\frac{1}{2} \boldsymbol{T}^{j} \times \boldsymbol{N}^{j} .
$$

Note that on $K_{\varepsilon}$ one has

$$
\boldsymbol{S}_{k}^{j} \times \boldsymbol{M}_{k}^{j}=\left(\boldsymbol{S}_{k}^{j}-\overline{\boldsymbol{R}}^{j}\right) \times \boldsymbol{M}_{k}^{j}+\overline{\boldsymbol{R}}^{j} \times\left(\boldsymbol{M}_{k}^{j}-\overline{\boldsymbol{R}}_{k}^{j}\right)+\overline{\boldsymbol{R}}^{j} \times \overline{\boldsymbol{R}}_{k}^{j}
$$

Therefore $\boldsymbol{S}_{k}^{j} \times \boldsymbol{M}_{k}^{j}$ tends uniformly to 0 on $K_{\varepsilon}$, so using Lemma 2.12 we obtain

$$
\left\|\boldsymbol{\psi}_{k}\right\|_{L^{\infty}\left(K_{\varepsilon} \cap H_{k}^{\varepsilon}, \mathbb{R}^{3}\right)} \rightarrow 0, \quad\left\|\exp \left(\mathbf{A}_{\psi_{k}}\right) \overline{\mathbf{R}}-\overline{\mathbf{R}}\right\|_{L^{\infty}\left(K_{\varepsilon} \cap H_{k}^{\varepsilon}, \mathbb{R}^{3 \times 3}\right)} \rightarrow 0 .
$$

Also note, because of (2.13), (2.14), (2.16), (2.17), it holds

$$
\begin{aligned}
& \boldsymbol{\psi}_{k} \rightarrow \boldsymbol{\psi} \text { weak* in } W^{1, \infty}\left(\Omega, \mathbb{R}^{3}\right), \\
& \left\|\boldsymbol{\psi}_{k}\right\|_{L^{\infty}\left(\mathbb{R}^{m}, \mathbb{R}^{3}\right)} \leq \frac{3}{2} C(\Omega)^{2}, \\
& \left\|\nabla \boldsymbol{\psi}_{k}\right\|_{L^{\infty}\left(\mathbb{R}^{m}, \mathbb{R}^{3 m}\right)} \leq 3 C(\Omega) c(m) \lambda_{2}(\varepsilon) .
\end{aligned}
$$

Using Lemma 2.1 one has

$$
\boldsymbol{\omega}_{k}^{i}(x)=\boldsymbol{\omega}^{i}(x)+\partial_{i}\left(\frac{1}{2} \overline{\boldsymbol{R}}^{j}(x) \times \overline{\boldsymbol{R}}_{k}^{j}(x)\right)+\boldsymbol{s}_{k}^{i}(x) \text { a.e. in } \Omega .
$$

Boundedness of $\partial_{i} \mathbf{M}_{k}$ on $H_{k}^{\varepsilon}$ and $\partial_{i} \mathbf{M}_{k}-\partial_{i} \overline{\mathbf{R}}_{k} \rightarrow 0$ strongly in $L^{\infty}\left(K_{\varepsilon}, \mathbb{R}^{3 \times m}\right), i=1, \ldots, m$ imply

$$
\left\|\partial_{i} \overline{\mathbf{R}}_{k}\right\|_{L^{\infty}\left(K_{\varepsilon} \cap H_{k}^{\varepsilon}, \mathbb{R}^{3 \times 3}\right)}<c(m) \lambda_{2}(\varepsilon)+1
$$

for almost every $k$. As $\partial_{i} \overline{\mathbf{R}}$ is continuous on $K_{\varepsilon}$ (and thus also bounded) and $\overline{\mathbf{R}}_{k} \rightarrow \overline{\mathbf{R}}$ strongly in $L^{\infty}\left(K_{\varepsilon}, \mathbb{R}^{3 \times 3}\right)$ we have that $\left\|s_{k}^{i}\right\|_{L^{\infty}\left(K_{\varepsilon} \cap H_{k}^{\varepsilon}, \mathbb{R}^{3}\right)} \rightarrow 0$. Now, because of uniform continuity of $W$ on compact sets and the boundedness of $\nabla \boldsymbol{\varphi}_{k}, \nabla \boldsymbol{g}_{k}, \boldsymbol{\omega}_{k}$ on $K_{\varepsilon} \cap H_{k}^{\varepsilon}$ and the fact that $\left\|\nabla \boldsymbol{\varphi}_{k}-\nabla \boldsymbol{z}_{k}\right\|_{L^{\infty}\left(K_{\varepsilon} \cap H_{k}^{\varepsilon}\right)} \rightarrow 0$ we have that

$$
\lim _{k \rightarrow \infty} \int_{K_{\varepsilon} \cap H_{k}^{\varepsilon}}\left|W\left(\nabla \boldsymbol{\varphi}_{k}, \overline{\mathbf{R}}_{k}, \boldsymbol{\omega}_{k}\right)-W\left(\nabla \boldsymbol{g}_{k}, \overline{\mathbf{R}}, \boldsymbol{\omega}+\nabla\left(\frac{1}{2} \overline{\boldsymbol{R}}^{j} \times \overline{\boldsymbol{R}}_{k}^{j}\right)\right)\right|=0 .
$$

In the same way we conclude

$$
\lim _{k \rightarrow \infty} \int_{K_{\varepsilon} \cap H_{k}^{\varepsilon}}\left|W\left(\nabla \boldsymbol{g}_{k}, \overline{\mathbf{R}}_{k}, \boldsymbol{\omega}+\nabla\left(\frac{1}{2} \overline{\boldsymbol{R}}^{j} \times \overline{\boldsymbol{R}}_{k}^{j}\right)\right)-W\left(\nabla \boldsymbol{g}_{k}, \overline{\mathbf{R}}, \boldsymbol{\omega}+\nabla \boldsymbol{\psi}_{k}\right)\right|=0
$$


By developing exponential function we have that

$$
\exp \left(\mathbf{A}_{\psi_{k}(x)}\right)=\mathbf{I}+\mathbf{A}_{\psi_{k}(x)}+\mathbf{r}_{k}(x)
$$

with the estimates for $i=1, \ldots, m$

$$
\begin{gathered}
\left\|\mathbf{r}_{k}\right\|_{L^{\infty}\left(\mathbb{R}^{m}, \mathbb{R}^{3 \times 3}\right)} \leq \exp \left(\frac{3}{2} C(\Omega)^{2}\right), \quad\left\|\partial_{i} \mathbf{r}_{k}\right\|_{L^{\infty}\left(\mathbb{R}^{m}, \mathbb{R}^{3 \times 3}\right)} \leq \exp \left(\frac{3}{2} C(\Omega)^{2}\right) 3 C(\Omega) c(m) \lambda_{2}(\varepsilon), \\
\left\|\mathbf{r}_{k}\right\|_{L^{\infty}\left(K_{\varepsilon} \cap H_{k}^{\varepsilon}, \mathbb{R}^{3 \times 3}\right)} \rightarrow 0, \quad\left\|\partial_{i} \mathbf{r}_{k}\right\|_{L^{\infty}\left(K_{\varepsilon} \cap H_{k}^{\varepsilon}, \mathbb{R}^{3 \times 3}\right)} \rightarrow 0 .
\end{gathered}
$$

Let us define

$$
\boldsymbol{\mu}_{k}^{i}=\frac{1}{2}\left(\exp \left(\mathbf{A}_{\psi_{k}}\right) \overline{\boldsymbol{R}}^{j}\right) \times \partial_{i}\left(\exp \left(\mathbf{A}_{\boldsymbol{\psi}_{k}}\right) \overline{\boldsymbol{R}}^{j}\right), \quad i=1, \ldots, m
$$

After an easy calculation using (2.21) we obtain

$$
\boldsymbol{\mu}_{k}=\boldsymbol{\omega}+\nabla \boldsymbol{\psi}_{k}+\mathbf{v}_{k}, \quad\left\|\mathbf{v}_{k}\right\|_{L^{\infty}\left(K_{\varepsilon} \cap H_{k}^{\varepsilon}, \mathbb{R}^{3 \times 3}\right)} \rightarrow 0 .
$$

Additionally, using (2.18), as before (see (2.19) and (2.20)), we conclude

$$
\lim _{k \rightarrow \infty} \int_{K_{\varepsilon} \cap H_{k}^{\varepsilon}}\left|W\left(\nabla \boldsymbol{g}_{k}, \overline{\mathbf{R}}, \boldsymbol{\omega}+\nabla \boldsymbol{\psi}_{k}\right)-W\left(\nabla \boldsymbol{g}_{k}, \exp \left(\mathbf{A}_{\psi_{k}}\right) \overline{\mathbf{R}}, \boldsymbol{\mu}_{k}\right)\right|=0
$$

Using (2.19), (2.20), (2.22) and nonnegativity of $W$ we have

$$
\begin{aligned}
\liminf _{k \rightarrow \infty} I\left(\boldsymbol{\varphi}_{k}, \overline{\mathbf{R}}_{k} ; \Omega\right) & \geq \liminf _{k \rightarrow \infty} I\left(\boldsymbol{\varphi}_{k}, \overline{\mathbf{R}}_{k} ;\left(K_{\varepsilon} \backslash A_{\varepsilon}\right) \cap H_{k}^{\varepsilon}\right) \\
& \geq \liminf _{k \rightarrow \infty} I\left(\boldsymbol{g}_{k}, \exp \left(\mathbf{A}_{\psi_{k}}\right) \overline{\mathbf{R}} ;\left(K_{\varepsilon} \backslash A_{\varepsilon}\right) \cap H_{k}^{\varepsilon}\right) .
\end{aligned}
$$

Since

$$
\begin{aligned}
\operatorname{meas}\left[\left(K_{\varepsilon} \backslash A_{\varepsilon}\right) \backslash H_{k}^{\varepsilon}\right] \leq & \sum_{i=1}^{3} \operatorname{meas}\left[\left(K_{\varepsilon} \backslash A_{\varepsilon}\right) \backslash H_{i, k}^{\lambda_{1}(\varepsilon)}\right]+\sum_{i, j=1}^{3} \operatorname{meas}\left[\left(K_{\varepsilon} \backslash A_{\varepsilon}\right) \backslash J_{i j, k}^{\lambda_{2}(\varepsilon)}\right] \\
& +\sum_{i, j=1}^{3} \operatorname{meas}\left[\left(K_{\varepsilon} \backslash A_{\varepsilon}\right) \backslash L_{i j, k}^{\lambda_{2}(\varepsilon)}\right] \leq 21 \min (\varepsilon, \delta),
\end{aligned}
$$


using the growth condition and the definition of $A_{\varepsilon}$ we have

$$
\begin{aligned}
I\left(\boldsymbol{g}_{k},\right. & \left.\exp \left(\mathbf{A}_{\psi_{k}}\right) \overline{\mathbf{R}},\left(K_{\varepsilon} \backslash A_{\varepsilon}\right) \backslash H_{k}^{\varepsilon}\right) \leq \int_{\left(K_{\varepsilon} \backslash A_{\varepsilon}\right) \backslash H_{k}^{\varepsilon}} K_{g}\left(1+\left\|\nabla \boldsymbol{g}_{k}\right\|^{r}+\left\|\boldsymbol{\mu}_{k}\right\|^{p}\right) \\
\leq & \int_{\left(K_{\varepsilon} \backslash A_{\varepsilon}\right) \backslash H_{k}^{\varepsilon}} K^{\prime}\left(1+\left\|\nabla \boldsymbol{g}_{k}\right\|^{r}+\sum_{i=1}^{m}\left\|\partial_{i}\left[\exp \left(\mathbf{A}_{\psi_{k}}\right) \overline{\mathbf{R}}\right]\right\|^{p}\right) \\
\leq & \int_{\left(K_{\varepsilon} \backslash A_{\varepsilon}\right) \backslash H_{k}^{\varepsilon}} 2^{p-1} K^{\prime}\left(1+\left\|\nabla \boldsymbol{g}_{k}\right\|^{r}+\sum_{i=1}^{m}\left\|\partial_{i} \exp \left(\mathbf{A}_{\psi_{k}}\right)\right\|^{p}+\|\boldsymbol{\omega}\|^{p}\right) \\
\leq & \int_{\left(K_{\varepsilon} \backslash A_{\varepsilon}\right) \backslash H_{k}^{\varepsilon}} 2^{2 p-2} K^{\prime \prime}\left(1+(2 c(m))^{r} \lambda_{1}(\varepsilon)^{r}+\exp \left(p \frac{3}{2} C(\Omega)^{2}\right) 3^{p} C(\Omega)^{p} c(m)^{p} \lambda_{2}(\varepsilon)^{p}+\|\boldsymbol{\omega}\|^{p}\right) \\
& \left(\operatorname{recall~that~} \lambda_{1}(\varepsilon)^{r}=\lambda_{2}(\varepsilon)^{p}\right) \\
\leq & K_{1}(m ; \Omega) \int_{\left(K_{\varepsilon} \backslash A_{\varepsilon}\right) \backslash H_{k}^{\varepsilon}}\left(1+\mid \lambda_{1}(\varepsilon)^{r}+\|\boldsymbol{\omega}\|^{p}\right) \\
\leq & K_{1}(m ; \Omega) \eta(21 \varepsilon)+K_{1}(m ; \Omega)\left(\sum_{i=1}^{3} \int_{\left(K_{\varepsilon} \backslash A_{\varepsilon}\right) \backslash H_{i, k}^{\lambda_{1}(\varepsilon)}}\left(M^{*} z_{k}^{(i)}\right)^{r}\right. \\
& \left.+\sum_{i, j=1}^{3} \int_{\left(K_{\varepsilon} \backslash A_{\varepsilon}\right) \backslash J_{i, k}^{\lambda_{2}(\varepsilon)}}\left(M^{*} M_{k}^{(i j)}\right)^{p}+\sum_{i, j=1}^{3} \int_{\left(K_{\varepsilon} \backslash A_{\varepsilon}\right) \backslash L_{i, k}^{\lambda_{2}(\varepsilon)}}\left(M^{*} S_{k}^{(i j)}\right)^{p}\right)=O(\varepsilon) .
\end{aligned}
$$

Therefore, taking into account (2.23) we obtain

$$
\liminf _{k \rightarrow \infty} I\left(\boldsymbol{\varphi}_{k}, \overline{\mathbf{R}}_{k} ; \Omega\right) \geq \liminf _{k \rightarrow \infty} I\left(\boldsymbol{g}_{k}, \exp \left(\mathbf{A}_{\psi_{k}}\right) \overline{\mathbf{R}} ; K_{\varepsilon} \backslash A_{\varepsilon}\right)-O(\varepsilon) .
$$

Because $\boldsymbol{g}_{k}, \boldsymbol{\psi}_{k}$ are bounded in $W^{1, \infty}\left(\Omega, \mathbb{R}^{3}\right)$ there exists a constant $C(\varepsilon, \Omega, m)$ such that for all $k$

$$
\left\|\boldsymbol{g}_{k}\right\|_{W^{1, \infty}\left(\Omega, \mathbb{R}^{3}\right)} \leq C(\varepsilon, \Omega, m), \quad\left\|\boldsymbol{\mu}_{k}(x)\right\| \leq C(\varepsilon, \Omega, m)(1+\|\omega(x)\|) \text { a.e. in } \Omega .
$$

Together with the growth condition of $W$ it implies that there exists $\Omega_{\varepsilon}^{\prime} \subset \Omega$ which contains $K_{\varepsilon} \backslash A_{\varepsilon}$ such that

$$
\left|I\left(\boldsymbol{g}_{k}, \exp \left(\mathbf{A}_{\psi_{k}}\right) \overline{\mathbf{R}} ; K_{\varepsilon} \backslash A_{\varepsilon}\right)-I\left(\boldsymbol{g}_{k}, \exp \left(\mathbf{A}_{\psi_{k}}\right) \overline{\mathbf{R}} ; \Omega_{\varepsilon}^{\prime}\right)\right|<\varepsilon
$$

Using nonnegativity of $W$ and Theorem 2.4 (it is easy to prove equiintegrability of $\boldsymbol{g}_{k}$ and $\exp \left(\mathbf{A}_{\psi_{k}}\right) \overline{\mathbf{R}}$, since $\left.\boldsymbol{g}_{k}, \boldsymbol{\psi}_{k} \in W^{1, \infty}\left(\Omega, \mathbb{R}^{3}\right)\right)$ and Lemma 2.12 we obtain

$$
\begin{aligned}
\liminf _{k \rightarrow \infty} I\left(\boldsymbol{\varphi}_{k}, \overline{\mathbf{R}}_{k} ; \Omega\right) & \geq \liminf _{k \rightarrow \infty} I\left(\boldsymbol{g}_{k}, \exp \left(\mathbf{A}_{\boldsymbol{\psi}_{k}}\right) \overline{\mathbf{R}} ; \Omega_{\varepsilon}^{\prime}\right)-O(\varepsilon)-\varepsilon \\
& \geq I\left(\boldsymbol{g}, \exp \left(\mathbf{A}_{\boldsymbol{\psi}}\right) \overline{\mathbf{R}} ; \Omega_{\varepsilon}^{\prime}\right)-O(\varepsilon)-\varepsilon
\end{aligned}
$$

Now we have to relate $\boldsymbol{g}$ and $\boldsymbol{\psi}$ with $\boldsymbol{\varphi}$ and $\boldsymbol{\phi}$ and 0 . Let us define

$$
G=\{x \in \Omega: g \neq \varphi \vee \psi \neq 0\} .
$$

On a subsequence one has $\boldsymbol{z}_{k}(x) \rightarrow \boldsymbol{\varphi}(x), \frac{1}{2} \boldsymbol{S}_{k}^{j}(x) \times \boldsymbol{M}_{k}^{j}(x) \rightarrow 0$ a.e. in $\Omega$. Therefore measure of

$$
\widetilde{G}=G \cap\left\{x \in \Omega: \boldsymbol{z}_{k}(x) \rightarrow \boldsymbol{\varphi}(x) \wedge \frac{1}{2} \boldsymbol{S}_{k}^{j}(x) \times \boldsymbol{M}_{k}^{j}(x) \rightarrow 0\right\}
$$


is the same as meas $(G)$. We shall prove that meas $(G) \leq 22 \varepsilon$. Let us assume the opposite, i.e. meas $(G)>22 \varepsilon$. Then by the definition of $H_{k}^{\varepsilon}$ it follows meas $\left(\mathbb{R}^{m} \backslash H_{k}^{\varepsilon}\right)<21 \varepsilon$. Therefore meas $\left(H_{k}^{\varepsilon} \cap \widetilde{G}\right) \geq \varepsilon$. Then by Lemma 2.7 at least on a subsequence we have

$$
\left(\bigcap_{h \in \mathbb{N}} H_{k_{h}}^{\varepsilon}\right) \bigcap \widetilde{G} \neq \emptyset
$$

For every $\widetilde{x}$ which belongs to the set on the left hand side one has

$$
\begin{aligned}
\boldsymbol{g}(\widetilde{x}) & =\lim _{h \rightarrow \infty} \boldsymbol{g}_{k_{h}}(\widetilde{x})=\lim _{h \rightarrow \infty} \boldsymbol{z}_{k_{h}}(\widetilde{x})=\boldsymbol{\varphi}(\widetilde{x}), \\
\boldsymbol{\psi}(\widetilde{x}) & =\lim _{h \rightarrow \infty} \boldsymbol{\psi}_{k_{h}}(\widetilde{x})=\lim _{h \rightarrow \infty} \frac{1}{2} \boldsymbol{S}_{k_{h}}^{j}(\widetilde{x}) \times \boldsymbol{M}_{k_{h}}^{j}(\widetilde{x})=0
\end{aligned}
$$

which is in contradiction with the definition of $G$. Thus we have

$$
\begin{aligned}
\liminf _{k \rightarrow \infty} I\left(\boldsymbol{\varphi}_{k}, \overline{\mathbf{R}}_{k} ; \Omega\right) & \geq I\left(\varphi, \overline{\mathbf{R}} ; \Omega_{\varepsilon}^{\prime} \backslash G\right)-O(\varepsilon)-\varepsilon \\
& \geq I(\varphi, \overline{\mathbf{R}} ; \Omega)-O(\varepsilon)-\varepsilon-\eta(24 \varepsilon) .
\end{aligned}
$$

Arbitrariness of $\varepsilon$ implies the claim.

Remark 2.14. The statement of the theorem holds for all $p, r \in[1, \infty]$, under the assumptions of Lemma 2.3. When $p=1$ or $r=1$ or $p=\infty$ or $r=\infty$ we can do the same analysis, but we do not change the sequence which is equiintegrable. For example when $p=1$ or $p=\infty$ we do not need to approximate $\overline{\mathbf{R}}_{k}$ (the construction of $\boldsymbol{\psi}_{k}$ and sets $J_{i j, k}^{\lambda_{2}(\varepsilon)}, L_{i j, k}^{\lambda_{2}(\varepsilon)}$ is unnecessary). The construction of $\boldsymbol{g}_{k}$ remains the same (if $r \in\langle 1, \infty\rangle$ ). Theorem 2.4 is then applied directly on $\left(\boldsymbol{g}_{k}\right)_{k},\left(\overline{\mathbf{R}}_{k}\right)_{k}$ to obtain $(2.24)$.

\section{REFERENCES}

[1] E. Acerbi and N. Fusco, Semicontinuity problems in the calculus of variations. Arch. Rational Mech. Anal. 86 (1984) $125-145$.

[2] I. Aganović, J. Tambača and Z. Tutek, Derivation and justification of the models of rods and plates from linearized threedimensional micropolar elasticity. J. Elasticity 84 (2006) 131-152.

[3] J.M. Ball, Convexity conditions and existence theorems in nonlinear elasticity. Arch. Rational Mech. Anal. 63 (1976/1977) $337-403$.

[4] P.G. Ciarlet, Mathematical elasticity, Volume I: Three-dimensional elasticity. North-Holland Publishing Co., Amsterdam (1988).

[5] E. Cosserat and F. Cosserat, Théorie des corps déformables. Librairie Scientifique A. Hermann et Fils (Translation: Theory of deformable bodies, NASA TT F-11 561, 1968), Paris (1909).

[6] B. Dacorogna, Direct methods in the calculus of variations. Second Edition, Springer (2008).

[7] A.C. Eringen, Microcontinuum Field Theories, Volume 1: Foundations and Solids. Springer-Verlag, New York (1999).

[8] I. Hlaváček and M. Hlaváček, On the existence and uniqueness of solution and some variational principles in linear theories of elasticity with couple-stresses. I. Cosserat continuum. Appl. Mat. 14 (1969) 387-410.

[9] J. Jeong and P. Neff, Existence, uniqueness and stability in linear Cosserat elasticity for weakest curvature conditions. Math. Mech. Solids (2008) DOI: 10.1177/1081286508093581.

[10] J. Jeong, H. Ramezani, I. Münch and P. Neff, Simulation of linear isotropic Cosserat elasticity with conformally invariant curvature. ZAMM Z. Angew. Math. Mech. (submitted).

[11] P.M. Mariano and G. Modica, Ground states in complex bodies. ESAIM: COCV (2008) DOI: 10.1051/cocv:2008036.

[12] N.G. Meyers, Quasi-convexity and lower semi-continuity of multiple variational integrals of any order. Trans. Amer. Math. Soc. 119 (1965) 125-149.

[13] P. Neff, On Korn's first inequality with nonconstant coefficients. Proc. R. Soc. Edinb. Sect. A 132 (2002) 221-243.

[14] P. Neff, Existence of minimizers for a geometrically exact Cosserat solid. Proc. Appl. Math. Mech. 4 (2004) 548-549.

[15] P. Neff, A geometrically exact Cosserat-shell model including size effects, avoiding degeneracy in the thin shell limit. Part I: Formal dimensional reduction for elastic plates and existence of minimizers for positive Cosserat couple modulus. Cont. Mech. Thermodynamics 16 (2004) 577-628.

[16] P. Neff, The Cosserat couple modulus for continuous solids is zero viz the linearized Cauchy- stress tensor is symmetric. $Z A M M$ Z. Angew. Math. Mech. 86 (2006) 892-912. 
[17] P. Neff, Existence of minimizers for a finite-strain micromorphic elastic solid. Proc. Roy. Soc. Edinb. A 136 (2006) $997-1012$.

[18] P. Neff, A finite-strain elastic-plastic Cosserat theory for polycrystals with grain rotations. Int. J. Eng. Sci. 44 (2006) 574-594.

[19] P. Neff, A geometrically exact planar Cosserat shell-model with microstructure. Existence of minimizers for zero Cosserat couple modulus. Math. Meth. Appl. Sci. 17 (2007) 363-392.

[20] P. Neff and K. Chelminski, A geometrically exact Cosserat shell-model for defective elastic crystals. Justification via $\Gamma$-convergence. Interfaces and Free Boundaries 9 (2007) 455-492.

[21] P. Neff and S. Forest, A geometrically exact micromorphic model for elastic metallic foams accounting for affine microstructure. Modelling, existence of minimizers, identification of moduli and computational results. J. Elasticity 87 (2007) $239-276$.

[22] P. Neff and J. Jeong, A new paradigm: the linear isotropic Cosserat model with conformally invariant curvature. $Z A M M Z$. Angew. Math. Mech. (submitted).

[23] P. Neff and I. Münch, Curl bounds Grad on SO(3). ESAIM: COCV 14 (2008) 148-159.

[24] W. Pompe, Korn's first inequality with variable coefficients and its generalizations. Commentat. Math. Univ. Carolinae 44 (2003) $57-70$.

[25] J. Tambača and I. Velčić, Derivation of a model of nonlinear micropolar plate. Ann. Univ. Ferrara Sez. VII Sci. Mat. 54 (2008) 319-333.

[26] J. Tambača and I. Velčić, Existence theorem for nonlinear micropolar elasticity. ESAIM: COCV (2008) DOI: 10.1051/cocv:2008065. 